\title{
eNeuro
}

Research Article: Methods/New Tools / Novel Tools and Methods

\section{Quantification of Neurite Degeneration with Enhanced Accuracy and Efficiency in an In Vitro Model of Parkinson's Disease}

https://doi.org/10.1523/ENEURO.0327-21.2022

Cite as: eNeuro 2022; 10.1523/ENEURO.0327-21.2022

Received: 23 July 2021

Revised: 21 January 2022

Accepted: 30 January 2022

This Early Release article has been peer-reviewed and accepted, but has not been through the composition and copyediting processes. The final version may differ slightly in style or formatting and will contain links to any extended data.

Alerts: Sign up at www.eneuro.org/alerts to receive customized email alerts when the fully formatted version of this article is published.

Copyright (c) 2022 Clements et al.

This is an open-access article distributed under the terms of the Creative Commons Attribution 4.0 International license, which permits unrestricted use, distribution and reproduction in any medium provided that the original work is properly attributed. 
Quantification of neurite degeneration with enhanced accuracy and efficiency in an in vitro model of Parkinson's disease

Rachel T Clements ${ }^{1 *}$, Lauren E Fuller ${ }^{1 *}$, Kyle R Kraemer ${ }^{2}$, Samantha A. Radomski ${ }^{1}$, Sarah Hunter-Chang ${ }^{3}$, Wesley C Hall ${ }^{1}$, Alborz A Kalantar ${ }^{1}$, and Bradley R Kraemer ${ }^{1}$

${ }^{*}$ These authors contributed equally to this work.

${ }^{1}$ From the Department of Biological Sciences, Eastern Kentucky University, Richmond, KY 40475

${ }^{2}$ From the Department of Psychology, Birmingham-Southern College, Birmingham, AL 35254

${ }^{3}$ From the Neuroscience Graduate Program, University of Virginia, Charlottesville, VA 22903

*Correspondence: Bradley R. Kraemer, Email: bradley.kraemer@eku.edu; Phone: 859-6221507; Address: 3238 Science Building, 521 Lancaster Ave., Richmond, KY 40475.

\begin{abstract}
Neurite degeneration is associated with early stages of neurodegenerative disorders such as Alzheimer's disease, Parkinson's disease, and amyotrophic lateral sclerosis. One method that is commonly used to analyze neurite degeneration involves calculation of a Degeneration Index (DI) following utilization of the Analyze Particles tool of ImageJ to detect neurite fragments in micrographs of cultured cells. However, DI analyses are prone to several types of measurement error, can be time consuming to perform, and are limited in application. Here, we describe an improved method for performing DI analyses. Accuracy of measurements was enhanced through modification of selection criteria for detecting neurite fragments, removal of image artifacts and non-neurite materials from images, and optimization of image contrast. Such enhancements were implemented into an ImageJ macro that enables rapid and fully automated $\mathrm{DI}$ analysis of multiple images. The macro features operations for automated removal of cell bodies from micrographs, thus expanding the application of DI analyses to use in experiments involving dissociated cultures. We present experimental findings supporting that, compared to the conventional method, the enhanced analysis method yields measurements with increased accuracy and requires significantly less time to perform. Furthermore, we demonstrate the utility of the method to investigate neurite degeneration in a cell culture model of PD by conducting an experiment revealing the effects of C-Jun N-terminal Kinase on neurite degeneration induced by oxidative stress in human mesencephalic cells. This improved analysis method may be used to gain novel insight into factors underlying neurite degeneration and the progression of neurodegenerative disorders.
\end{abstract}

\title{
Significance Statement
}

Neurite degeneration is a cellular event associated with the early stages of multiple types of neurodegenerative disorders. Molecular factors that regulate neurite degeneration remain poorly understood, and existing methods for studying neurite degeneration have limited application and efficiency. Here, we identify modifications to a widely used procedure for analyzing neurite degeneration that reduce measurement error. Such methodological enhancements were incorporated into an ImageJ macro, thereby facilitating rapid and completely automated analysis 
of neurite degeneration using large sets of micrographs. Using a cell culture model of Parkinson's disease, we demonstrate that the macro facilitates more accurate and efficient neurite degeneration measurements while expanding the suitability of the analysis method for experiments involving dissociated cultures.

\section{Introduction}

Neurite degeneration is a cellular process in which axons or dendrites undergo progressive dysfunction and morphological changes, leading to a loss in neurite integrity. Neurite degeneration is an evolutionarily conserved process that is critical for the proper establishment of neural networks during the development of the central nervous system (Neukomm and Freeman, 2014). However, the process is also triggered in response to various forms of injury such as mechanical damage or exposure to drugs used for chemotherapy (Salvadores et al., 2017). Furthermore, neurite degeneration is associated with the progression of a variety of agerelated neurodegenerative disorders, including Alzheimer's disease, Parkinson's disease (PD), Huntington's disease, and amyotrophic lateral sclerosis. Interestingly, for all such neurodegenerative disorders, accumulating evidence suggests that neurite degeneration is a key, early-stage event that precedes neuronal death or onset of clinical symptoms (SerranoPozo et al., 2011; Adalbert and Coleman, 2013; Marangoni et al., 2014; Suzuki et al., 2020). Thus, studies exploring factors that regulate this cellular process may reveal novel targets for treating a variety of a neurodegenerative conditions.

Investigating neurite degeneration is particularly important for understanding PD, a disease characterized by the accumulation of protein aggregates containing a-synuclein, progressive loss of dopaminergic neurons in the substantia nigra pars compacta (SNpc), and the development of a variety of motor and cognitive symptoms (Aarsland et al., 2017). Evidence from postmortem and human imaging studies indicates that $\alpha$-synuclein aggregates are more abundant in neuronal fibers than in the cell bodies, and degradation of dopaminergic axons occurs to a greater extent than loss of dopaminergic neurons in the SNpc during the period leading to clinical onset (Tagliaferro and Burke, 2016). While the molecular interactions that contribute to the progressive deterioration of dopaminergic neurites remain incompletely understood, recent evidence indicates that neurite degeneration can be mediated by events independent of signaling cascades underlying neuronal death (Fukui, 2016; Geden and Deshmukh, 2016). Thus, studies which directly evaluate physiological underpinnings of neurite degeneration are critical to the development of therapies that impede early PD progression.

In response to the evidence of a central role for neurite degeneration in the progression of neurodegenerative disorders, a variety of methods have been developed to measure neurite degeneration using micrographs of cultured neurons. For example, multiple different methods have been established to measure neurite degeneration using software to detect decreased neurite number or length (Ofengeim et al., 2012; Johnstone et al., 2018). However, since such methods do not directly evaluate neurite fragmentation, interpretation of results to determine the impact of a variable on neurite degeneration can be confounded by potential effects of the variable on neurite outgrowth. Certain research groups have also measured neurite degeneration by subjectively scoring images of neurites based on morphological features and the degree of apparent fragmentation (Zhai et al., 2003; Nakamura et al., 2016; Krishtal et al., 2017). However, this alternative method is time consuming and may introduce investigator error. 
One method that facilitates objective and direct measurement of neurite fragmentation involves use of the Analyze Particles algorithm of ImageJ to measure the area of neurite fragments in micrographs of cultured cells. The measurements are used to calculate a Degeneration Index (DI), a scale between $0.0-1.0$ reflecting the degree of neurite degeneration (Sasaki et al., 2009). While DI analyses can be used to detect varying degrees of neurite fragmentation, the accuracy of measurements is commonly limited by several sources of error, and application of the method has been primarily limited to explant cultures or cells grown in compartmentalized microfluidic devices. Moreover, DI analyses are time consuming when extrapolated to numerous images sets. Thus, we sought to overcome these limitations by modifying the DI analysis procedure to facilitate greater accuracy and efficiency, while expanding applicability.

In the present study, we identify several sources of error commonly associated with DI analyses and report methodological revisions that reduce the various forms of error. We incorporate the procedural enhancements in an ImageJ macro termed ANDI (Automated Neurite Degeneration Index) to completely automate DI analyses from large image sets. Operation of the ANDI macro only requires software and files that are open source and freely available. Measurements obtained using ANDI benefit from increased accuracy while substantially reducing the time required to perform the analyses. Moreover, we demonstrate how the new analysis method can be applied to perform accurate and rapid measurement of neurite degeneration in a cell culture model of PD involving dissociated cells grown without compartmentalization. Collectively, these methodological enhancements may lead to novel insight into factors underlying an important cellular process associated with neurodegeneration.

\section{Materials and Methods}

\section{Cell Culture}

Cultures of Lund Human Mesencephalic (LUHMES) cells were established in Lab Tek II eight-well chamber slides coated with poly-L-ornithine and fibronectin, poly-D-lysine and laminin, all four substrates, or no substrates, as indicated. To coat with poly-L-ornithine and fibronectin, slides were incubated overnight at room temperature with a solution containing 200 $\mu \mathrm{g} / \mathrm{mL}$ poly-L-ornithine (Sigma-Aldrich, St. Louis, MO, Cat\# P3655). Following washes with sterile water (Avantor, Radnor, Pennsylvania), the slides were incubated with $2 \mu \mathrm{g} / \mathrm{mL}$ fibronectin (Sigma-Aldrich, Cat\# F0895) for three hours at $37^{\circ} \mathrm{C}$. Slides were then washed with sterile phosphate-buffered saline (PBS) (Corning Life Sciences, Cat\# 21-040-CV) and allowed to air-dry. To coat with poly-D-lysine and laminin, slides were incubated at room temperature with a solution containing $100 \mathrm{ng} / \mathrm{mL}$ poly-D-lysine (Sigma-Aldrich, Cat\#P7280) and $10 \mu \mathrm{g} / \mathrm{mL}$ laminin (Corning, Corning, NY, Cat\# 354232). Following an overnight incubation, the slides were washed with sterile PBS and allowed to air dry. To coat with all four substrates, the aforementioned procedures were performed sequentially. LUHMES cells (ATCC, Manassas, VA, RRID:CVCL_B056) were plated in the coated chamber slides at the indicated densities in differentiation medium consisting of Dulbecco's Modified Eagle Medium with Nutrient Mixture F12 (DMEM/F12) (Gibco, Waltham, MA, Cat\# 11330057) supplemented with $2 \mathrm{mM}$ glutamine (VWR), 1\% (v/v) N-2 supplement, $1 \mu \mathrm{g} / \mathrm{mL}$ tetracycline (Sigma-Aldrich, Cat\# 87128-25G), $1 \mathrm{mM}$ N6,2'-O-Dibutyryladenosine 3',5'-cyclic monophosphate (db-cAMP) (Enzo Life Sciences, Farmingdale, NY, Cat\# BML-CN125-0100), and $2 \mathrm{ng} / \mathrm{mL}$ glial cell line-derived neurotrophic factor (GDNF) (R\&D Systems, Cat\# 212-GD-010). Except where otherwise indicated, all cultures were plated at a density of 115,000 cells per well. A $50 \%$ volume media replacement 
was performed every 48 hours prior to treatment. To prevent genetic drift, cell stocks that had been passaged six or fewer times were used for all experiments.

Experiments involving primary cultures of sympathetic neurons were approved by the Animal Care and Use Committee at Eastern Kentucky University (Fig. 6A - C and 6F-G) or the University of Virginia (Fig. 6D and 6E). Superior cervical ganglia were dissected from postnatal day $0-6 \mathrm{C} 56 \mathrm{BI} / 6$ mice, incubated at 37 degrees Celsius in an enzymatic dissociation buffer, washed with growth media to remove proteolytic enzymes, and triturated. Cell suspensions were plated at a density of 7,000 cells per well in Lab Tek II eight-well chamber slides. Media was changed at DIV 1 , followed by media replacement every other day. The media was supplemented with cytosine arabinofuranoside (Ara-C, Millipore-Sigma) on DIV 1-3 to prevent the growth of non-neuronal cells, followed by removal of Ara-C from the media for at least 24 hours prior to treatment as indicated. For experiments conducted at Eastern Kentucky University (Fig. 6A - 6C and 6F - 6G), the chamber slides were coated with $200 \mu \mathrm{g} / \mathrm{mL}$ poly-Lornithine (Sigma-Aldrich, Cat\# P3655), $2 \mu \mathrm{g} / \mathrm{mL}$ fibronectin, $100 \mathrm{ng} / \mathrm{mL}$ poly-D-lysine (SigmaAldrich, Cat\#P7280) and $10 \mu \mathrm{g} / \mathrm{mL}$ laminin (Corning, Corning, NY, Cat\# 354232) prior to use, and dissociation was performed via 30 minute incubation in buffer consisting of Dulbecco's modified Eagle's medium (DMEM, ThermoFisher, Cat\# 11960-044) containing 0.025\% trypsin (Corning Life Sciences, Durham, NC, Cat\# 25-053-Cl) and 0.3\% collagenase (Worthington Biochemical, Cat\# LS004176). Growth media for such experiments consisted of DMEM supplemented with 10\% fetal bovine serum (Gibco, Cat\# 26140-079), 2 mM L-glutamine (Corning, Cat\# 25-005-Cl), and $40 \mathrm{ng} / \mathrm{mL}$ nerve growth factor (Fisher Scientific, Cat\# 13-257019. $5 \mu \mathrm{M}$ Ara-C (Sigma-Aldrich) was used for removal of non-neuronal cells. For experiments conducted at the University of Virginia (Fig. 6D and 6E), cells were plated on chamber slides coated with $100 \mathrm{ng} / \mathrm{mL}$ poly-D-Lysine (Millipore Sigma) and $10 \mu \mathrm{g} / \mathrm{mL}$ Laminin (Gibco), and dissociation was performed via 20 minute incubation in buffer consisting of Dulbecco's modified Eagle's medium with Nutrient Mixture F-12 (DMEM/F12) containing BSA (10 mg/mL, Sigma Aldrich), collagenase type $2(4 \mathrm{mg} / \mathrm{mL}$, Worthington Biochemical), deoxyribonuclease 1 (Millipore Sigma), and hyaluronidase (Sigma Aldrich). Growth media for such experiments consisted of DMEM supplemented with $10 \%$ fetal bovine serum (Gibco), $1 \mathrm{U} / \mathrm{mL}$ penicillin/streptomycin (Gibco), and $45 \mathrm{ng} / \mathrm{mL}$ of NGF (purified from salivary glands). $10 \mu \mathrm{M}$ Ara$\mathrm{C}$ was used for removal of non-neuronal cells.

\section{Cell Treatments}

Differentiated LUHMES cells were exposed to the indicated concentrations of 6-OHDA (Sigma-Aldrich, Cat\# 162957) or vehicle solution on their fifth day in differentiation medium. Stocks of $6-\mathrm{OHDA}$ were prepared in chilled PBS containing $0.02 \%$ ascorbate (Sigma-Aldrich, Cat\# A5960), and aliquots were stored in light-protected vials under inert gas at $-80^{\circ} \mathrm{C}$. After 24 hours of treatment, the cells were fixed by incubation in PBS containing $4 \%$ paraformaldehyde for 25 minutes at room temperature. To determine the role of c-Jun N-terminal Kinase (JNK) in neurite degeneration induced by oxidative stress, cultures were pretreated for 1 hour with $10 \mu \mathrm{M}$ SP600125 (Sigma-Aldrich, Cat\# S5567) or vehicle solution prior to complete replacement of media with media containing 7.5 $\mu \mathrm{M}$ 6-OHDA, $7.5 \mu \mathrm{M}$ 6-OHDA and $10 \mu \mathrm{M}$ SP600125, or vehicle solutions. Stocks of SP600125 were prepared by dissolving the inhibitor in sterile dimethylsulfoxide (DMSO) and frozen aliquots were stored in light-protected vials filled with inert gas at $-20^{\circ} \mathrm{C}$. Treatments were performed such that cells were exposed to no greater than $0.1 \%$ DMSO. 
Primary cultures of sympathetic neurons were treated on DIV 5. For experiments involving neurite degeneration induced by hydrogen peroxide, the cultures were exposed to $500 \mu \mathrm{M}$ hydrogen peroxide (Sigma Aldrich, Cat\# H1009-100ML) or vehicle solution consisting of sterile water (Cytiva, Cat\# SH30529.02) for 24 hours. For experiments involving NGF withdrawal, SCG cultures either received a media replacement with growth media or were subjected to NGF deprivation. SCG cultures to be deprived of NGF were rinsed three times with NGF-free growth media and then incubated in NGF-free growth media for 80 hours without media changes. After treatment, the cells were fixed by incubation in $4 \%$ paraformaldehyde in PBS for 30 minutes, followed by removal of the paraformaldehyde by rinsing with PBS.

\section{Staining and Fluorescence Microscopy}

For experiments with LUHMES cells, as well as the experiments involving sympathetic neurons associated with Fig. 6A-C and 6F-G, eight-well chamber slides containing fixed cells were permeabilized with $0.1 \%$ Triton-X-100 in PBS and blocked for one hour with $10 \%$ normal goat serum in PBS containing $0.1 \%$ Triton-X-100. The slides were then incubated with primary antibody specific for BIII-tubulin (Covance, Princeton, NJ; 1:1000, RRID:AB_2313773) in blocking solution at $4^{\circ} \mathrm{C}$ overnight. Subsequently, the slides were washed with PBS, incubated with a secondary antibody coupled to Alexa Fluor 488 (Thermo Fisher Scientific; 1:500, RRID: AB_142495) for 90 minutes at room temperature, and subjected to additional washes. For experiments involving tyrosine hydroxylase staining, the slides were subjected to an additional incubation in PBS containing $10 \%$ normal goat serum, $0.1 \%$ Triton-X-100, and antibody specific for tyrosine hydroxylase (Sigma-Aldrich, 1:400, AB152) at 4 degrees Celsius overnight. The slides were then washed with PBS, incubated with secondary antibody coupled to Alexa Fluor 568 (Thermo Fisher Scientific; 1:500, RRID: AB_1500889), and subjected to additional PBS washes. Following completion of immunolabeling for Blll-tubulin or tyrosine hydroxylase, slides were incubated with $5 \mu \mathrm{g} / \mathrm{mL}$ 4',6'-diamidino-2-phenylindole (DAPI) (Cat\# D9542) in PBS for 5 minutes. Slides were then washed with PBS, and coverslips were mounted using Fluoromount $\mathrm{G} B$ mounting medium (Southern Biotech, Birmingham, AL, Cat\# 0100-01).

For experiments conducted at the University of Virginia (Fig. 6D and 6E), the slides were blocked for 1 hour by incubation at room temperature in buffer consisting of $5 \%$ normal goat serum (Life Technologies) and $0.0005 \%$ Triton X-100 in PBS, followed by incubation in primary antibody solution containing mouse anti-mouse Tuj1 (1:1000, Covance) overnight at 4 degrees Celsius. Subsequently, slides were rinsed with PBS, incubated for 1 hour in goat anti-mouse IgG AlexaFluor 488 (1:800, Invitrogen) and $0.1 \mathrm{ug} / \mathrm{mL}$ Hoescht 33342 (Invitrogen) in blocking buffer, subjected to additional PBS rinses, and allowed to dry. The slides were mounted with Fluoromount-G (Southern Biotech, Cat\# 0100-01) and stored at room temperature.

Slides containing LUHMES cells were visualized with a 20X objective of a Nikon Eclipse II Ti-U Inverted Microscope System with a Lumencor Mira 4-Channel LED light source. $1280 \mathrm{x}$ 1024 pixel images were captured using a Nikon DS-Qi1Mc 5 MP CCD Camera and NIS Elements 3.22.15 (Build 738). Except for where otherwise indicated, all phase contrast images were captured using light intensity and exposure time settings that produced an average background intensity of 165 in 8-bit images. To reduce background shading or other inconsistencies, prior to each microscopy session an image of an empty well was captured and used for background correction during LUHMES cell imaging. Slides containing sympathetic neurons cultured at were imaged using a Zeiss LSM 800 microscope system (Fig. 6A-C and Fig. 6F - 6G) or using a Zeiss LSM 980 microscope (Fig. 6D and 6E). Images were captured at 
20X magnification and $1024 \times 1024$ resolution using Zen software (Carl Zeiss Microscopy, Jena, Germany).

\section{Cell Viability Analysis}

Differentiated LUHMES cell cultures were established on Lab Tek II eight-well chamber slides that were coated as indicated with various substrates. A $50 \%$ media change was performed every 48 hours, and the cells were fixed with 4\% PFA in PBS on the sixth day of differentiation. Slides containing fixed cells were subjected to staining with DAPI, and images of nuclear staining were captured with a Nikon Eclipse II Ti-U Inverted Microscope System and Nikon DS-Fi1 5 MP CCD Camera. Cells were scored as healthy or unhealthy by a blinded investigator based on the appearance of the nuclei. Cells with decreased nuclear area or nuclear fragmentation were scored as unhealthy. The number of healthy cells per image was scored for at least five fields of view per condition in each experiment.

\section{Degeneration Index Measurements}

Traditional DI measurements were performed using a Fiji Is Just Image (FIJI) package containing ImageJ $1.52 \mathrm{p}$ via a method involving widely cited parameters for $\mathrm{DI}$ analysis (Shin et al., 2012; Di Stefano et al., 2015; Sasaki et al., 2016; Loreto et al., 2020; Shin and Cho, 2020). 8-bit images of differentiated LUHMES cells with well-separated axon tracts were subjected to binarization using the default binarization algorithm, with binary options configured to produce an image with black cells in the foreground and white background. The freehand drawing tool was used to manually trace all image regions featuring cell bodies, and all black pixels were converted to white in the traced regions. The Measure tool of ImageJ was applied to measure the total black area of the binarized images exclusively featuring neurites. The Analyze Particle tool was then applied using a size setting of $20-10,000$ pixels and a circularity setting of $0.2-$ 1.0 to detect neurite fragments. The summed area of all neurite fragments was then divided by the total black area to calculate the degeneration index of the image.

For experiments related to optimization of size parameters for identification of neurite fragments, circularity criteria of $0.2-1.00$ were used for all particle measurements. The Particle Remover plugin (version 2004/02/09), developed by Wayne Rasband, was obtained from https://imagej.nih.gov/. Where indicated, application of Particle Remover plugin using size parameters of $0-9$ pixels and circularity parameters of $0.0-1.0$ was performed to remove small, non-neurite objects from binarized images obtained from phase-contrast micrographs. Similarly, size parameters of $0-4$ pixels and circularity parameters of $0-1.0$ were used to remove small objects from binarized images obtained from fluorescence micrographs representing BIII-tubulin immunostaining. Unless otherwise indicated, prior to $\mathrm{DI}$ analysis all phase contrast images were subjected to contrast enhancement by using Image $J$ to apply a Lookup Table (LUT) with a minimum of 90 and maximum of 205.

\section{Measurement of Artificial Fragmentation}

Cultures containing healthy LUHMES cells were fixed with $4 \%$ PFA on their $6^{\text {th }}$ day of differentiation and immunolabeled for BIII-tubulin. Using a 20X objective, $1280 \times 1024$ pixel phase contrast micrographs and fluorescence micrographs, each depicting identical fields of view, were captured from randomly sampled regions. A total of ten sets of images were captured from nine separate cultures. ImageJ was used to generate binarized copies of the original phase-contrast and fluorescence micrographs, as well as copies of phase-contrast 
micrographs that were binarized after contrast enhancement via application of an LUT with a minimum of 90 and maximum of 205. For each set of images, a blinded investigator identified a small, $150 \times 150$ pixel region of the original phase contrast micrograph featuring exclusively healthy and intact neurites. The region size and coordinates were then stored in the ROI manager of ImageJ and used to perform DI measurements in analogous regions of binarized images from the same image set. The DI measurements were performed using the traditional method featuring fragment size parameters of $0-20$ pixels. Since all regions measured contained exclusively intact neurons, any fragments detected were considered artificial fragments.

\section{Automated Neurite Degeneration Index (ANDI) Macro}

The ANDI macro was written using ImageJ Macro language. The macro features a series of operations that enable the user to select paths for three directories, one directory containing images of neurons immunolabeled for a neuronal marker such as BIII-tubulin, a second directory containing corresponding images depicting nuclear staining, and a third directory designated for saving result files. The fragment areas and total neurite areas of the images in the selected directories are then calculated via a series of ImageJ operations. In brief, an image depicting nuclear labeling is opened, subjected to contrast enhancement, and binarized. The particle remover plugin is used to remove small, non-nuclear artifacts from the binarized image, and the binarized image is subjected to a series of dilations and erosions. $\mathrm{A}$ corresponding image of cells immunolabeled for Blll-tubulin (or other neuronal marker) is opened and subjected to contrast enhancement. The binarized image depicting enlarged cell nuclei is then subtracted from the image featuring cells immunolabeled for Blll-tubulin, thus generating an image featuring exclusively neurites. The neurite image is then binarized and subjected to removal of particles between $0-4$ pixels. Neurite fragment area is calculated by applying the Analyze Particle algorithm to measure particles with a circularity between $0.2-1.0$ and size between $5-10,0000$ pixels. Total neurite area is calculated by applying the Measure tool to calculate the total black area. An image depicting the binarized neurite area with highlighted fragments is saved to a directory designated by the user, along with .csv files reporting the fragmented neurite area and total neurite area measurements.

In addition to the aforementioned operations, several additional operations are included in the macro to support ease-of-use and limit user error. These include functions that remove scale information from images and ensure that measurements are obtained in pixel units; configure the ImageJ color settings and binary options for appropriate image subtraction and binarization; verify that the selected directories for images of BIII-tubulin staining and DAPI staining contain an equal number of images; close previously used images to enhance processing time; clear results from previous analyses prior to performance of new analyses; and prevent errors associated with .ini file creation by Windows 10 .

For experiments involving analyses of sympathetic neurons, the ANDI macro was revised to include an operation that presents a dialog box enabling the user to control the number of times that binarized images of nuclei will be dilated. The version of ANDI featuring this revision was termed ANDI v1.1. Changing the value in the dialog box to a higher number increases the soma removal size, while changing the value to a lower number decreases the soma removal size. The default value is 12 , which is the number of dilations used for all experiments involving LUHMES cells. A value of 16 was used to remove larger cell body regions during analyses of sympathetic neurons. 
The ANDI Macro is open source and freely available for use or modification via the following url: https://github.com/kraemerb/kraemerlab. Accurate operation of ANDI has been verified using multiple personal computers featuring the Windows 10 operating system. An image set featuring LUHMES cell cultures exhibiting various degrees of neurite degeneration is also available at the aforementioned web addressed and can be used to investigate accurate execution of the macro by other types of computers. The web address also provides access to a protocol describing how to use the macro.

\section{Time Estimates}

To evaluate the time required to manually perform DI measurements, phase contrast micrographs depicting LUHMES cells that were treated with vehicle solution, $2.5 \mu \mathrm{M} 6-\mathrm{OHDA}$, 5.0 $\mu \mathrm{M}$ 6-OHDA, or $7.5 \mu \mathrm{M} 6$-OHDA were subjected to DI scoring using previously-established methods(Kraemer et al., 2014). Each image was subjected to contrast enhancement and binarization. The freehand tool of ImageJ was then used to digitally remove all depicted cell bodies from the binarized images. The Analyze Particle tool was used to measure fragments with a circularity between $0.2-1.0$ and a size between $20-10,000$ pixels, and the Measure tool was used to determine the total neurite area. Values pertaining to fragmented neurite area and total neurite area were transferred to a spreadsheet in Microsoft Excel, and a mathematical operation in Excel was used to calculate the DI scores. After an image was opened with ImageJ, the time required to manually perform each of these procedures was measured. Average analysis times were calculated from 36 total images. The images were obtained from 9 independent cell culture preparations, analyses were performed using one image per treatment condition from each cell culture preparation. To determine the speed of DI measurements performed using the ANDI macro, the same image sets were used to determine the time required to open the macro in ImageJ, run the macro operations, transfer the data to an Excel spreadsheet, and calculate the DI.

\section{Statistics}

Quantitative comparisons are presented as box and whisker plots, where the middle line represents the median, boxes extend to the 25 and 75 percentiles, and whiskers depict the 5 and 95 percentiles. Means were statistically compared in GraphPad Prism 9.1.2 by repeated measures ANOVA with post-hoc comparisons using a Šidák correction. Normality for quantitative data was tested using the Shapiro-Wilk test in IBM SPSS 26 . When the assumption of normality was violated (see Fig. 1C and 3D), group comparisons were conducted via nonparametric Friedman test with Dunn's test performed for post-hoc comparisons. Correlational analyses are depicted as scatterplots and include lines of best fit. Such relationships were analyzed using Pearson product moment correlations or Spearman's rho when the assumption of normality was violated. Normality of residuals for correlational analysis was assessed through visual inspection of corresponding Q-Q plots. Pearson product moment correlation coefficients were compared to one another using Steiger's asymptotic z-test (Steiger, 1980; Lee, 2013). No outliers were excluded. Statistical significance was accepted at $p<0.05$. P-values are rounded to four decimal places and reported throughout the main text. Effect sizes and $95 \%$ confidence intervals are also reported for parametric analyses, with effect sizes described throughout the main text and confidence intervals listed in Table-1. 


\section{Results}

\section{Establishment of a Cell Culture Model of Neurite Degeneration Associated with Parkinson's} Disease

To develop a method for investigating neurite degeneration in a cell culture model of Parkinson's disease, we used cultured Lund Human Mesencephalic (LUHMES) cells, a population of conditionally immortalized, human mesencephalic cells. Upon differentiation, LUHMES cells become post-mitotic, adopt a gene expression profile characteristic of mature dopaminergic neurons, and develop a neuronal morphology with neurites that extend up to 500 $\mu \mathrm{m}$ in length (Fig. 1A) (Lotharius et al., 2005; Scholz et al., 2011; Kraemer et al., 2021). Due to the superior optical qualities of glass compared to plastic, the cultures were established on glass slides containing eight media chambers. Based on previous reports (Lotharius et al., 2005; Scholz et al., 2011; Kurowska et al., 2014), we initially attempted to establish cultures on chamber slides coated with poly-L-ornithine (PLO) and fibronectin; however, such cultures exhibited adhesion deficits and low viability. As a result, we tested whether coating with additional substrates would improve the viability of LUHMES cell cultures established on glass surfaces. LUHMES cell cultures were established on slides that were either uncoated or coated with PLO and fibronectin, poly-D-lysine (PDL) and laminin, or a combination of all four substrates. Analysis of viable nuclei by fluorescent microscopy revealed that slide coating significantly affected the viability of the cultures [Friedman $X 2(3)=18, p<0.0001$ ], with post-hoc multiple comparison analyses revealing that slides coated with a combination of all four aforementioned substrates yield a significantly higher rate of viable cells when compared with slides only coated with PLO and fibronectin [ $p=0.0437$ ] (Fig. 1B and 1C).

After identifying the optimal coating substrates for culturing LUHMES cells on glass slides, we evaluated the effects of cell density on the suitability of the cultures for neurite degeneration measurements. LUHMES cells were cultured in differentiation medium with plating densities of 57,500 cells per well, 115,000 cells per well, or 230,000 cells per well, and on their fifth day of differentiation the cells were fixed with $4 \%$ paraformaldehyde and imaged by phase contrast microscopy. A density of 57,500 cells per well resulted in cultures with poorly networked neurites and irregular neurite morphology, while cultures established with a plating density of 230,000 cells, though viable, contained neurite densities too high for accurate neurite measurement. The intermediate cell density of 115,000 cells per well provided high viability and well-separated axon tracts conducive to accurate neurite degeneration measurements (Fig. 1D).

To model PD-associated neurite degeneration in vitro, we exposed differentiated LUHMES cells to 6-hydroxydopamine (6-OHDA), a widely used neurotoxin that promotes oxidative stress and degeneration of catecholinergic neurons (Bove and Perier, 2012). Exposure of differentiated LUHMES cells to 6-OHDA promoted a dose-dependent increase in neurite degeneration, with $5.0 \mu \mathrm{M}$ 6-OHDA and 7.5 $\mu \mathrm{M}$ 6-OHDA inducing moderate and severe neurite degeneration, respectively (Fig. 2F). To quantify neurite degeneration in cultures of LUHMES cells exposed to 6-OHDA, we used a widely cited method in which phase-contrast images of cultures are binarized, cell bodies in the images are digitally removed, and the particle analyzer algorithm of ImageJ is applied to measure the area of neurite fragments. The fragmented neurite areas are then summed and divided by the total neurite area to determine a degeneration index (DI) (Fig. 2A - 2E) (Sasaki et al., 2009; Kraemer et al., 2014; Di Stefano et al., 2015; Hill et al., 2018; Geisler et al., 2019). As expected, exposure of differentiated LUHMES cells to 6-OHDA caused a dose-dependent increase in the DI $[F(3,24)=119.8$, 
$\left.p=0.0001, R^{2}=0.9374\right]$ (Fig. 2G). However, during the analyses of cells exposed to 6-OHDA we observed several issues that appeared to negatively impact the accuracy and efficiency of DI measurements. Thus, we sought to optimize this common method for performing DI measurements - which we henceforth refer to as the traditional DI method - to generate a protocol facilitating more effective measurement of neurite fragmentation.

\section{Reducing Artificial Fragmentation Associated with Micrograph Binarization.}

While using the traditional DI method, we frequently observed an issue in which intact neurites in phase-contrast images appeared fragmented in their corresponding binarized image (Fig. 3A). This artificial fragmentation primarily affected neurites with low prominence. Thus, we investigated whether the issue could be reduced by procedures that enhance image contrast. To improve image contrast, we first captured images of neurites using a variety of light intensities to determine the level of image brightness that would produce optimal contrast. Images captured with the optimal light intensity were then subjected to further contrast enhancement by using ImageJ to apply a Lookup Table with minimum and maximum intensity values of 90 and 205, respectively. Incorporation of these contrast enhancements provided a moderately more accurate representation of the neurites in binarized images generated from phase-contrast images (Fig. 3B). Nevertheless, quantitative analysis of binarized images of healthy neurites indicated that the contrast enhancements did not significantly decrease artificial fragmentation [ $p=0.9999]$ (Fig. 3D). Thus, we next investigated whether use of immunofluorescence imaging would produce images with superior contrast and thereby facilitate production of more accurate binarized neurite images with reduced artificial fragmentation. The slides described in Figure 2, featuring fixed LUHMES cells exposed to various concentrations of 6-OHDA, were immunostained for the cytoskeletal protein BIII-tubulin, imaged using fluorescence microscopy, and analyzed by a blinded investigator for artificial fragmentation (Fig. 3C). Quantitative analysis of binarized images obtained from fluorescence micrographs and corresponding phase-contrast micrographs revealed that the type of microscopy did affect levels of artificial fragmentation [Friedman $x^{2}(3)=15.44, p<0.0001$ ]. Specifically, fluorescence micrographs produced binarized images with significantly reduced artificial fragmentation compared to those from phase-contrast micrographs $[p=0.0016]$ (Fig. 3D). As a result, images of healthy cultures yielded significantly lower DI scores when florescence micrographs were used rather than phase-contrast micrographs $[F(1,8)=131.4$, $p=0.0001, R^{2}=0.1442$ ] (Fig. 3E). Moreover, a notable overall trend is that the fold change between mean DI values associated with healthy (vehicle-treated) cultures and cultures exhibiting neurite degeneration $(5.0 \mu \mathrm{M}$ - or $7.5 \mu \mathrm{M}$ - 6-OHDA-treated) were greater when measured using fluorescence micrographs than when obtained from phase contrast micrographs (Fig. 3E). This occurred despite fluorescence micrographs of cultures with moderate neurite degeneration (induced by exposure to $5 \mu \mathrm{M} 6-\mathrm{OHDA}$ ) having significantly lower DI scores than corresponding phase images due to the reduction in artificial fragmentation $[p=0.0001, d=0.6312]$.

\section{Optimizing Parameters for Fragmented Neurite and Total Neurite Detection}

The traditional method for performing DI measurements involves use of the ImageJ Analyze Particles plugin with size parameters of $20-10,000$ pixels to detect neurite fragments. However, we observed that a large proportion of neurite fragments were undetected by the particle analyzer when using these widely cited parameters (Shin et al., 2012; Di Stefano et al., 2015; Sasaki et al., 2016; Loreto et al., 2020; Shin and Cho, 2020) to measure neurite 
degeneration in phase contrast micrographs of LUHMES cells exposed to 6-OHDA, generated as described in Fig. 2. Thus, we reanalyzed images from that dataset using different minimum size limits for particle detection to determine the optimal parameters for identification of neurite fragments. From qualitative assessment of binarized images generated from phase-contrast micrographs, size parameters of $5-10,000$ pixels enhanced the detection of neurite fragments, but was also associated with misidentification of background noise, image artifacts, or other small non-neurite material as neurite fragments. Compared with the widely used parameters of $20-10,000$ pixels, however, parameters of $10-10,000$ pixels enhanced detection of neurite fragments without a noticeably large increase in false positives (Fig. 4A, left). Similar analyses were also performed with binarized images obtained from fluorescence micrographs of LUHMES cells immunolabeled for BIII-tubulin. Particle analyzer size parameters of $5-10,000$ pixels facilitated the most sensitive detection of neurite fragments and, since the fluorescence micrographs featured less small debris, such parameters did not noticeably increase misidentification of non-neurite material as neurite fragment (Fig. 4A, right).

The traditional method for performing DI measurements, by utilizing particle analyzer size parameters of $20-10,000$ pixels, excludes objects smaller than 20 pixels from measurements of neurite fragments. However, such small objects are typically erroneously included in the total neurite area measurements, since the traditional DI measurement method involves calculating total neurite area by summing all black pixels in the binarized images. To overcome this issue, we used the Particle Remover plugin for ImageJ, written by ImageJ developer Wayne Rasband, to remove small, non-neurite objects from binarized images. Application of this plugin while performing DI measurements using phase contrast micrographs of LUHMES cells exposed to 6-OHDA, generated as described in Fig. 2, resulted in a significant increase in DI measurements across all concentrations of neurotoxin exposure $[F(1,8)=83.43$, $\left.p=0.0001, R^{2}=0.0416\right]$. Furthermore, a significant interaction indicated that the increase in DI due to use of the particle remover became stronger at higher concentrations $[F(1.881,15.05)=$ 59.97, $p=0.0001, R^{2}=0.0094$ ] (Fig. 4B and $4 \mathrm{C}$ ). We also imaged the same slides of cells by fluorescence microscopy and assessed the effects of small particle removal on DI measurements obtained from fluorescence micrographs. While small particle removal provides DI values that are theoretically more accurate, such values on average did not significantly differ from those obtained without use of the particle remover plugin when measured using fluorescence micrographs $\left[F(1,8)=2.613, p=0.1446, R^{2}=0.0013\right]$ (Fig. 4D).

\section{Automation of Degeneration Index Measurements from Fluorescence Micrographs}

Performance of DI measurements using the traditional method requires tedious and time-consuming image processing, use of the freehand tool to digitally remove individual cell bodies, and measurement of fragmented neurite and total neurite areas from each image. Thus, we sought to enhance the efficiency of the method by writing a macro that fully automates DI analyses. To automate soma removal, the macro, titled ANDI (Automated Neurite Degeneration Index), executes the binarization of images of cell nuclei, labeled via the nuclear stain 4',6diamidino-2-phenylindole (DAPI), followed by modification of the binarized image such that thresholded nuclear regions are enlarged to encompass the entire region of the soma. Such images with binarized and enlarged nuclei are then subtracted from corresponding fluorescence micrographs of neurons immunolabeled for BIII-tubulin, generating an image exclusively featuring neurites (Fig. 5A-5B). To adjust for variable nuclear sizes caused by nuclear shrinkage or fragmentation associated with neurodegeneration, the binarized images of nuclei 
are enlarged via a combination of dilations and erosions that combine nuclear fragments and expand binarized regions to occupy an area larger than the somas of differentiated LUHMES cells. Images exclusively containing neurites are then binarized and subjected to DI analysis via a series of automated functions (Fig. $5 C-5 D$ ). To evaluate the accuracy of the macro operations associated with soma removal from images, we compared the DI scores obtained following manual removal of cell bodies from fluorescence micrographs using the freehand tool of ImageJ (as previously depicted in Fig. 4D), to DI scores generated from analyses of identical images following automated cell body removal using the soma removal operations featured in the ANDI macro. Our analyses revealed that DI scores calculated using automated cell body removal correlated nearly identically with DI measurements obtained from images subjected to manual soma removal $[r(34)=0.980, p<0.0001]$ (Fig. 5E).

To increase the accuracy of the analyses, we incorporated several of the previously described adjustments that enhance DI measurements into ANDI. The macro features contrast enhancement operations, use of the Particle Remover plugin to remove non-neurite matter from neurite images and non-nuclear matter from images of nuclei, and detection of neurite fragments using size parameters of $5-10,000$ for the Analyze Particles plugin. We hypothesized that, collectively, the use of fluorescence microscopy, contrast enhancement operations, removal of small objects with the particle remover, and optimized parameters for particle detection would contribute to more accurate measurements of neurite degeneration. To test this hypothesis, the phase contrast images of LUHMES cells exposed to various concentrations of 6-OHDA, generated as described in Fig. 2, were analyzed by blind and subjective scoring using a value range between $0.0-1.0$, with values representing the apparent proportion of fragmented neurite area. We then evaluated correlation between the subjective scores and DI measurements obtained via the traditional method, as well as correlation between the subjective scores and DI measurements obtained using the ANDI macro. While DI measurements obtained using the traditional method correlated with subjective scores $\left[r^{2}=\right.$ 0.7850, $p<0.0001$ ] (Fig. 5F), the ANDI macro yielded measurements that correlated much more strongly with subjective ratings $\left[r^{2}=0.978, p<0.0001\right]$ (Fig. $5 G$ ). Direct statistical comparisons revealed that scores obtained via the ANDI macro correlated significantly stronger with subjective ratings than do scores obtained via the traditional method $[z=6.626, p<0.0001]$ (Fig. 5F-G). Further analyses revealed that the macro facilitates performance of DI measurements with robust and significant improvement in time efficiency. DI analyses performed using the ANDI macro required an average time of 12.75 seconds per image analyzed, compared to an average time of 5.27 minutes per image analyzed via the traditional method [Friedman $\mathrm{X} 2(1)=9, p=0.0027$ ]. Altogether, these results indicate that ANDI yields rapid generation of DI measurements that, compared to scores obtained via the traditional method, more closely approximate the degeneration that investigators perceive from qualitative analysis of neurite images.

After determining the accuracy and efficiency of the ANDI macro for measuring neurite degeneration in differentiated LUHMES cells exposed to 6-OHDA, we evaluated the suitability of the macro for experiments involving other cell culture models. A pilot study was first conducted to assess the accuracy of ANDI in performing DI measurements using micrographs of primary sympathetic cultures immunolabeled for $\beta$ III-tubulin and DAPI. Interestingly, however, the cell bodies of sympathetic neurons are larger than those of differentiated LUHMES cells, and thus the operations in ANDI related to automated soma removal did not remove the entirety of the soma (data not shown). To overcome this issue, we revised ANDI to feature a dialog box 
enabling users to choose the size of the soma removal by controlling the number of times that binarized micrographs of nuclei are dilated. This version of ANDI, version 1.1, was then used to perform DI measurements from micrographs depicting healthy, vehicle-treated sympathetic neurons or degenerating sympathetic neurons exposed to hydrogen peroxide. Images of neurons exposed to hydrogen peroxide yielded significantly higher DI scores compared to images of vehicle-treated neurons $\left[F(1,3)=71.2, p=0.0035, R^{2}=0.9301\right]$ (Fig. 6A and 6C). Moreover, we compared these DI scores that were obtained using ANDI to DI measurements obtained from the same image set following manual removal of cell bodies from fluorescence micrographs using the freehand tool of ImageJ. There was no significant difference in DI scores calculated following manual soma removal compared to scores obtained using ANDI $[F(1,3)=$ 0.1082, $p=0.7639, R^{2}=0.0000$ ] (Fig. $6 \mathrm{~A}-6 \mathrm{C}$ ). Post-hoc tests also confirmed that DI scores obtained using ANDI were not significantly different both in the case of untreated neurons $(p=0.0972)$ and in the case of neurons exposed to hydrogen peroxide $(p=0.9986)$. Furthermore, DI scores produced from ANDI correlated nearly identically with DI scores obtained following manual removal of cell bodies from corresponding micrographs $[r(34)=0.991, p<0.0001]$. These data demonstrate that ANDI is suitable for measuring neurite degeneration in cultures of primary neurons, with soma removal operations that the user can customize to match different neuron types. Additionally, the detection of neurite fragments in cultures exposed to hydrogen peroxide demonstrates the applicability of the macro for use in experiments involving neurite degeneration induced by sources other than 6-OHDA. To further verify the use of ANDI in detecting neurite degeneration associated with various causes, we also used the macro to measure neurite degeneration in micrographs of healthy sympathetic cultures or cultures subjected to NGF withdrawal. As expected, images of cultures subjected to NGF withdrawal yielded significantly higher DI values compared to images of healthy cultures $[F(1,2)=287, p=$ $0.0035, R^{2}=0.958$ ] (Fig. $6 \mathrm{D}$ and $6 \mathrm{~F}$ ). Collectively, these findings demonstrate that ANDI can be used to detect neurite degeneration associated with a variety of biological contexts.

Since staining for tyrosine hydroxylase $(\mathrm{TH})$ is commonly used to visualize catecholinergic neurons, we also evaluated the suitability of micrographs depicting TH staining for measuring neurite degeneration using ANDI. Cultures of healthy sympathetic neurons treated with vehicle solution or degenerating sympathetic neurons exposed to hydrogen peroxide were fixed and subjected to immunofluorescence labeling for $\mathrm{TH}$ and BIII-tubulin, as well as counterstaining for DAPI. Neurite images depicting staining for TH or BIII-tubulin, and corresponding images of nuclei featuring DAPI labeling, were then captured at similar fields of view. ANDI was executed, and the images featuring TH-staining or BIII-tubulin staining were selected as the neurite images. Our analyses revealed that scores obtained using images of $\mathrm{TH}$-staining correlated very strongly with scores obtained from images of BIII-tubulin staining $[r(28)=0.962, p<0.0001]$ (Fig. 6F and 6G). These data indicate that accurate DI measurements can be obtained by ANDI using alternative staining procedures that label the entirety of neurites, such as immunolabeling for $\mathrm{TH}$.

To demonstrate the utility of the improved method for performing DI measurements in a scientific experiment, we used the new method to assess the role of c-Jun N-terminal Kinase (JNK) in oxidative stress-induced neurite degeneration in human mesencephalic cells. Differentiated LUHMES cells were pretreated for one hour with the JNK inhibitor SP600125 or vehicle solution. The cells were then treated for 24 hours with 6-OHDA to induce oxidative stress or with vehicle solution. Fixed cells were subjected to immunolabeling for BIII-tubulin and DAPI staining, and ANDI was used to obtain DI measurements from fluorescence micrographs. 
While cultures lacking pretreatment with the JNK inhibitor exhibited neurite degeneration following exposure to 6-OHDA, JNK inhibition resulted in a marked and significant reduction in 6 -OHDA-induced neurite degeneration $[p=0.0001, d=2.0209]$ (Fig. 7). These findings support a key role for JNK signaling in neurite degeneration induced by oxidative stress in human mesencephalic cells, and importantly, demonstrate the utility of our new method for generating scientific discoveries by facilitating the rapid and accurate measurement of neurite degeneration.

\section{Discussion}

Neurite degeneration is associated with a variety of neuropathological conditions, yet the molecular mechanisms underlying degradation of neurites remain incompletely understood. Methods facilitating accurate and efficient analysis of neurite degeneration are essential to the successful identification of novel factors regulating this important cellular event. In the present study, we reveal multiple sources of error associated with a commonly used method for quantifying neurite degeneration. We report experimental data supporting procedural modifications that can be implemented to reduce DI analysis error, and such modifications are incorporated into a new ImageJ macro to provide a tool for rapid, accurate, and objective DI analyses using open source and free software. Moreover, we demonstrate how the improved method can be applied to measure neurite degeneration in a cell culture model of PD, a disorder in which axonal fragmentation in dopaminergic neurons is an early-stage event that precedes eventual neuronal loss (Tagliaferro and Burke, 2016).

In the present study we demonstrate the utility of the ANDI macro in measuring neurite degeneration in a cell culture model of PD consisting of differentiated LUHMES cells exposed to 6-OHDA. We report experimental findings supporting the optimal substrates upon which the cultures should be established, as well as demonstrate the plating density that is most appropriate for neurite degeneration analyses. Use of this model system to study neurite degeneration has several advantages. The cells develop neurites that are well-networked and typically more than $500 \mu \mathrm{m}$ in length, and the cultures are susceptible to neurodegeneration induced by classic, neurotoxin models of PD such as 6-OHDA, 1-methyl-4-phenylpyridinium ion (MPP+), and rotenone (Lotharius et al., 2005; Zhang et al., 2014; Smirnova et al., 2016; Kraemer et al., 2021). Furthermore, the human origin of LUHMES cells increases the translatability of findings associated with the model, and the conditional immortalization facilitates rapid generation and propagation of cultures (Scholz et al., 2011). Thus, coupled with automated analyses performed using ANDI, LUHMES cells can be used to make novel discoveries related to PD-associated neurite degeneration while facilitating considerably higher throughput compared to non-automated analyses performed with primary neuronal cultures.

Numerous research groups have performed DI analyses using phase contrast images to gain insight into factors that regulate neurite degeneration (Press and Milbrandt, 2009; Kraemer et al., 2014; Di Stefano et al., 2015; Hill et al., 2018; Geisler et al., 2019). Here, we report an important drawback associated with this widely used method for measuring neurite degeneration: that binarization of phase contrast images causes a significant proportion of intact neurites to appear fragmented. While the level of contrast that may be achieved when capturing micrographs varies depending upon the microscope system available to investigators, this issue is apparently pervasive among various research groups, as a DI of 0.2 is commonly accepted as a threshold above which cultures are considered to have degenerating axons, and most scientific reports featuring DI measurements from phase contrast micrographs have reported DI 
scores for healthy neurons between $0.1-0.3$ (Sasaki et al., 2009; Di Stefano et al., 2015; Sasaki et al., 2016; Hill et al., 2018; Loreto et al., 2020). Our DI analyses obtained from fluorescence micrographs, as well as subjective scores from blinded investigators, indicate that $10-20 \%$ neurite fragmentation is not common to healthy neuron cultures. Rather, such cultures exhibit only $2.5 \%$ neurite fragmentation on average. Thus, our findings underscore the need for methods that improve the accuracy of neurite degeneration analyses by reducing this common and significant source of measurement error.

While the majority of studies involving DI measurements have used phase contrast images to perform the neurite degeneration analyses, several research groups have recently performed DI measurements using images of neurons immunolabeled for cytoskeletal filaments such as neurofilament medium polypeptide or B-tubulin (Wakatsuki et al., 2011; Li et al., 2017; PeaseRaissi et al., 2017; Hernandez et al., 2018; Sundaramoorthy et al., 2020). However, which form of microscopy is best suited for neurite degeneration measurements has remained unclear, as investigations have been needed to understand whether changes in the localization of these cytoskeletal proteins during neurite degeneration appropriately model total changes in neurite morphology, as well as to evaluate the degree to which DI measurements obtained with fluorescence micrographs correlate with similar measurements obtained from phase contrast micrographs. Here, we demonstrate that fluorescence micrographs depicting BIII-tubulin staining not only accurately represent neurite fragmentation, but also facilitate more accurate DI measurements due to their superior contrast and decreased susceptibility to artificial fragmentation upon binarization. Thus, our results highlight the utility of using BIII-tubulin staining to measure neurite degeneration.

Among the published studies involving DI analyses to measure neurite degeneration, the reported size and circularity parameters used to detect neurite fragments have considerably varied. For example, a recent study involving assessment of neurite degeneration in cultured hippocampal neurons utilized size parameters of 4-900 pixels (Li et al., 2017), while a study involving cultured DRG neurons used detection parameters of $0-10,000$ pixels (Wakatsuki et al., 2011). One potential reason for such variation is that the efficacy of particle analysis size parameters in detecting neurite fragments varies depending upon the resolution of the image, since the size parameters for particle detection are associated with pixel units. Unfortunately, most published studies involving $\mathrm{DI}$ analyses do not include a description of the image resolution used for the analyses. Thus, the establishment of standard analysis parameters via a protocol that fully discloses important details such as the recommended image resolution has been needed. Here, we report that the most commonly-cited parameters for neurite fragment detection, 20 - 10,000 pixels (Shin et al., 2012; Di Stefano et al., 2015; Sasaki et al., 2016; Loreto et al., 2020; Shin and Cho, 2020), result in large proportions of neuron fragments being undetected. Reducing the minimum size criterion for neurite fragment detection enhances the sensitivity of fragment detection, while simultaneously increasing the number of image artifacts and non-neurite debris that are falsely detected as neurite fragments. In search for parameters that would best balance detection sensitivity and false-positivity rates, we identified analysis parameters of $10-10,000$ as providing the most accurate detection of neurite fragments in binarized images obtained from phase contrast micrographs, while parameters of $5-10,000$ facilitate the most accurate detection of neurite fragments in binarized images obtained from fluorescence micrographs. While these determinations were made using an image resolution of $1280 \times 1024$, such parameters should also enable accurate neurite fragment detection in images captured at other resolutions featuring a vertical size of 1024 pixels, such as the 
common full screen resolution of $1024 \times 1024$, since neurons depicted in such images would be of similar pixel size.

Numerous research groups have used a minimum size criterion when configuring particle analysis parameters to avoid small, non-neurite debris from being included in neurite fragment measurements (Shin et al., 2012; Cosker et al., 2013; Di Stefano et al., 2015; Sasaki et al., 2016; Li et al., 2017; Loreto et al., 2020; Shin and Cho, 2020; Sundaramoorthy et al., 2020). However, such studies have erroneously included small, non-fragment matter in the measurements of total neurite area, since such measurements are obtained by summing the area of all black pixels in the image. Here, we demonstrate the utility of the Particle Remover plugin to remove small, non-fragment debris from the image prior to DI analysis. Utilization of the plugin significantly increased the sensitivity of neurite degeneration measurements obtained from phase contrast images. Thus, our results highlight the value of the Particle Remover plugin for investigators performing DI analyses using phase contrast micrographs. Interestingly, fluorescence micrographs featured less small, non-neurite debris, and thus, a lower size criterion of 5 pixels could be used for fragment detection. While particle removal of non-neurite debris less than 5 pixels in size did not significantly affect DI measurements obtained from fluorescence micrographs, such operations provide values that are theoretically more accurate and therefore were included in ANDI.

Since DI analyses must be performed using images exclusively featuring neurites, application of the analysis method has primarily been limited to use with culture systems amenable to removal of cell bodies, such as explant cultures in which cell bodies can be physically excised (Catenaccio et al., 2017; Shin and Cho, 2020) or cultures in microfluidic devices facilitating segregation of soma and axon compartments (Cosker et al., 2013; Pease-Raissi et al., 2017; Tan et al., 2018). Alternatively performing DI analyses with dissociated cultures can be achieved through tedious and time consuming labor associated with digital removal of cell bodies from micrographs (Kraemer et al., 2014). In the present study, we reveal that ANDI enables performance of DI measurements from micrographs featuring LUHMES cells that are dissociated and evenly distributed across a $2 \mathrm{~d}$ culture system. Our findings also demonstrate that ANDI facilitates a 24-fold decrease in time required to perform the analyses, and the operations in ANDI related to soma removal yield DI analysis results that correlate $98 \%$ with similar analyses performed following manual soma removal using the freehand tool of ImageJ. Thus, the macro substantially increases the efficiency of the analyses by performing automated soma removal via a process that does not sacrifice accuracy, and such operations expand the suitability of the method for analyzing dissociated cultures with mosaically-distributed cell bodies.

In addition to facilitating automated cell body removal from images, ANDI features a several revisions to the traditional method for performing DI analysis, including use of fluorescence micrographs, revised particle analysis parameters, and utilization of the particle remover to remove small, non-neurite debris from images. Our findings indicate that these optimizations collectively yield DI values that more closely approximate values obtained through blinded and subjected scoring. Thus, in addition to substantially reducing the time required to perform the analyses, ANDI facilitates DI measurements that, compared to the traditional method, more accurately reflect the neurite degeneration that investigators reportedly observe from qualitative image analysis. 
One drawback of the traditional method for performing $\mathrm{DI}$ analyses is that such analyses require training in use of Image $J$ so that the user is familiar with a tedious set of ImageJ operations. Moreover, to perform the analyses in an efficient manner, further training is required for the user to perform batch analyses or to write scripts to semi-automate execution of particular steps. By fully automating the analysis, ANDI facilitates DI measurements in only a few steps related to downloading and opening the macro. To increase user-friendliness, the macro includes code to provide an interface for selecting directories containing images to be analyzed, as well as to facilitate output of result images and data tables to a directory of the user's choosing. Moreover, the script contains numerous features designed to prevent measurement inaccuracies or user mistakes, including automated clearing of previous ImageJ results; removal of image scale information to ensure measurements are accurately performed using pixel units; appropriate configuration of Image J color settings, measurement settings, and particle analyzer parameters; error message presentation if users erroneously select directories containing unequal numbers of images depicting BIII-tubulin staining and DAPI staining; log descriptions indicating analysis progress; and coding to prevent bugs associated with automated ini file generation by Windows 10. Altogether, these features make $\mathrm{DI}$ analyses more user friendly, which may foster interest in studies related to neurite degeneration.

To demonstrate its utility in an experiment, we used ANDI to evaluate the contributions of JNK to neurite degeneration in LUHMES cell cultures exposed to 6-OHDA. JNK is a stress-activated kinase that has been reported as key to neurite degeneration in peripheral neurons of nonprimate origin (Shin et al., 2012), but further studies are needed to understand the role of the kinase in neurite degeneration in mesencephalic human cells. Here, we demonstrate that inhibition of JNK significantly protects human mesencephalic cells from neurite degeneration associated with oxidative stress. Such findings not only reveal an important role for JNK in neurite degeneration associated with a model of human disease, but also exemplify the utility of ANDI for performing automated and objective image analysis to make new scientific discoveries.

While ANDI was initially designed to facilitate rapid and accurate measurement of neurite degeneration in cultures of differentiated LUHMES cells exposed to 6-OHDA, our findings also indicate that the macro can be used to detect neurite degeneration induced by other causes, such as exposure to hydrogen peroxide or neurotrophin withdrawal. Additionally, version 1.1 of the macro features a dialog box enabling investigators to customize the soma removal operations for neurons of specific sizes. Our data also supports that staining methods other than immunolabeling for BIII-tubulin can be used for accurate measurement of neurite degeneration. Although we recommend the use of pilot studies to verify the accuracy of DI measurements when using ANDI with cell types or staining methods that have not formerly been tested, these findings support that the macro is a generally versatile tool for measuring neurite degeneration in cultured neurons. Since ANDI has not been optimized for measuring neurite degeneration in tissue, we currently only recommend its use for analyzing neurite degeneration associated with cultured cells. However, the code for ANDI is publicly available and thus may also be useful for investigators interested in modifying the script to suit in vivo studies or other applications.

In conclusion, this article presents data revealing common sources of error associated with a widely used method for quantifying neurite degeneration. Experimental approaches were used to improve the method, and such enhancements were incorporated into a free and open-source ImageJ macro that can be used to perform rapid, accurate, and objective analysis of 
fluorescence micrographs for neurite degeneration. Our findings reveal the efficacy of ANDI for quantifying neurite degeneration in micrographs depicting a cell culture model of Parkinson's disease, as well as provide proof of principle that the macro can detect neurite degeneration associated with other biological contexts. The user-friendly macro will reduce the time required for investigators to learn to quantify neurite fragmentation, while increasing the throughput and accuracy of studies evaluating factors underlying neurite degeneration. The ANDI macro is available at the following url: https://github.com/kraemerb/kraemerlab.

\section{Acknowledgements}

The authors thank Dr. Clinton Hasenour for comments and suggestions about the manuscript. We also thank Sarah Sparks for assistance with the artificial fragmentation analyses and preparation of online resources related to ANDI. We acknowledge the Keck Center for Cellular Imaging for the usage of the Zeiss 980 multiphoton Airyscan microscopy system (PI- AP; NIH0D025156).

\section{Funding}

This work was supported by the Kentucky Biomedical Infrastructure Network (KBRIN) [ULRF13-1493C-04 and ULRF-13-1493B-01]; the National Institute of General Medical Sciences NIGMS [8P20GM103436-14], and the Battelle-EKU Science Scholars Program. The content is solely the responsibility of the authors and does not necessarily represent the official views of the National Institutes of Health.

\section{Data Availability}

Additional data pertaining to the present article will be shared upon reasonable request. Inquiries should be submitted to Dr. Bradley Kraemer via the following email address: bradley.kraemer@eku.edu

\section{Code Accessibility}

The code/software described in the paper is freely available online at https://github.com/kraemerb/kraemerlab. The code is available as Extended Data.

\section{Conflicts of Interest}

The authors declare no competing financial interests. 
References

Aarsland D, Creese B, Politis M, Chaudhuri KR, Ffytche DH, Weintraub D, Ballard C (2017) Cognitive decline in Parkinson disease. Nature reviews Neurology 13:217-231.

Adalbert R, Coleman MP (2013) Review: Axon pathology in age-related neurodegenerative disorders. Neuropathol Appl Neurobiol 39:90-108.

Bove J, Perier C (2012) Neurotoxin-based models of Parkinson's disease. Neuroscience 211:51-76.

Catenaccio A, Llavero Hurtado M, Diaz P, Lamont DJ, Wishart TM, Court FA (2017) Molecular analysis of axonal-intrinsic and glial-associated co-regulation of axon degeneration. Cell Death Dis 8:e3166.

Cosker KE, Pazyra-Murphy MF, Fenstermacher SJ, Segal RA (2013) Target-derived neurotrophins coordinate transcription and transport of bclw to prevent axonal degeneration. J Neurosci 33:5195-5207.

Di Stefano M et al. (2015) A rise in NAD precursor nicotinamide mononucleotide (NMN) after injury promotes axon degeneration. Cell Death Differ 22:731-742.

Fukui K (2016) Reactive oxygen species induce neurite degeneration before induction of cell death. J Clin Biochem Nutr 59:155-159.

Geden MJ, Deshmukh M (2016) Axon degeneration: context defines distinct pathways. Curr Opin Neurobiol 39:108-115.

Geisler S, Doan RA, Cheng GC, Cetinkaya-Fisgin A, Huang SX, Hoke A, Milbrandt J, DiAntonio A (2019) Vincristine and bortezomib use distinct upstream mechanisms to activate a common SARM1-dependent axon degeneration program. JCI Insight 4.

Hernandez DE, Salvadores NA, Moya-Alvarado G, Catalan RJ, Bronfman FC, Court FA (2018) Axonal degeneration induced by glutamate excitotoxicity is mediated by necroptosis. $J$ Cell Sci 131. 
Hill CS, Menon DK, Coleman MP (2018) P7C3-A20 neuroprotection is independent of Wallerian degeneration in primary neuronal culture. Neuroreport 29:1544-1549.

Johnstone AD, Hallett RM, de Leon A, Carturan B, Gibon J, Barker PA (2018) A novel method for quantifying axon degeneration. PLoS One 13:e0199570.

Kraemer BR, Snow JP, Vollbrecht P, Pathak A, Valentine WM, Deutch AY, Carter BD (2014) A role for the p75 neurotrophin receptor in axonal degeneration and apoptosis induced by oxidative stress. J Biol Chem 289:21205-21216.

Kraemer BR, Clements RT, Escobedo CM, Nelson KS, Waugh CD, Elliott AS, Hall WC, Schemanski MT (2021) c-Jun N-terminal Kinase Mediates Ligand-independent p75(NTR) Signaling in Mesencephalic Cells Subjected to Oxidative Stress. Neuroscience 453:222-236.

Krishtal J, Bragina O, Metsla K, Palumaa P, Tougu V (2017) In situ fibrillizing amyloid-beta 1-42 induces neurite degeneration and apoptosis of differentiated SH-SY5Y cells. PLoS One 12:e0186636.

Kurowska Z, Brundin P, Schwab ME, Li JY (2014) Intracellular Nogo-A facilitates initiation of neurite formation in mouse midbrain neurons in vitro. Neuroscience 256:456-466.

Lee IAaP, K.J. (2013) Calculation for the test of the difference between two dependent correlations with one variable in common [Computer software].

Li LS, Lu YL, Nie J, Xu YY, Zhang W, Yang WJ, Gong QH, Lu YF, Lu Y, Shi JS (2017) Dendrobium nobile Lindl alkaloid, a novel autophagy inducer, protects against axonal degeneration induced by Abeta25-35 in hippocampus neurons in vitro. CNS Neurosci Ther 23:329-340.

Loreto A, Hill CS, Hewitt VL, Orsomando G, Angeletti C, Gilley J, Lucci C, Sanchez-Martinez A, Whitworth AJ, Conforti L, Dajas-Bailador F, Coleman MP (2020) Mitochondrial impairment activates the Wallerian pathway through depletion of NMNAT2 leading to SARM1-dependent axon degeneration. Neurobiol Dis 134:104678. 
Lotharius J, Falsig J, van Beek J, Payne S, Dringen R, Brundin P, Leist M (2005) Progressive degeneration of human mesencephalic neuron-derived cells triggered by dopaminedependent oxidative stress is dependent on the mixed-lineage kinase pathway. $J$ Neurosci 25:6329-6342.

Marangoni M, Adalbert R, Janeckova L, Patrick J, Kohli J, Coleman MP, Conforti L (2014) Agerelated axonal swellings precede other neuropathological hallmarks in a knock-in mouse model of Huntington's disease. Neurobiol Aging 35:2382-2393.

Nakamura S, Nakanishi A, Takazawa M, Okihiro S, Urano S, Fukui K (2016) lonomycin-induced calcium influx induces neurite degeneration in mouse neuroblastoma cells: analysis of a time-lapse live cell imaging system. Free Radic Res 50:1214-1225.

Neukomm LJ, Freeman MR (2014) Diverse cellular and molecular modes of axon degeneration. Trends Cell Biol 24:515-523.

Ofengeim D, Shi P, Miao B, Fan J, Xia X, Fan Y, Lipinski MM, Hashimoto T, Polydoro M, Yuan J, Wong ST, Degterev A (2012) Identification of small molecule inhibitors of neurite loss induced by Abeta peptide using high content screening. J Biol Chem 287:8714-8723.

Pease-Raissi SE, Pazyra-Murphy MF, Li Y, Wachter F, Fukuda Y, Fenstermacher SJ, Barclay LA, Bird GH, Walensky LD, Segal RA (2017) Paclitaxel Reduces Axonal Bclw to Initiate IP3R1-Dependent Axon Degeneration. Neuron 96:373-386 e376.

Press C, Milbrandt J (2009) The purine nucleosides adenosine and guanosine delay axonal degeneration in vitro. J Neurochem 109:595-602.

Salvadores N, Sanhueza M, Manque P, Court FA (2017) Axonal Degeneration during Aging and Its Functional Role in Neurodegenerative Disorders. Frontiers in neuroscience 11:451.

Sasaki Y, Vohra BP, Lund FE, Milbrandt J (2009) Nicotinamide mononucleotide adenylyl transferase-mediated axonal protection requires enzymatic activity but not increased levels of neuronal nicotinamide adenine dinucleotide. J Neurosci 29:5525-5535. 
Sasaki Y, Nakagawa T, Mao X, DiAntonio A, Milbrandt J (2016) NMNAT1 inhibits axon degeneration via blockade of SARM1-mediated NAD(+) depletion. Elife 5.

Scholz D, Poltl D, Genewsky A, Weng M, Waldmann T, Schildknecht S, Leist M (2011) Rapid, complete and large-scale generation of post-mitotic neurons from the human LUHMES cell line. J Neurochem 119:957-971.

Serrano-Pozo A, Frosch MP, Masliah E, Hyman BT (2011) Neuropathological alterations in Alzheimer disease. Cold Spring Harb Perspect Med 1:a006189.

Shin JE, Cho Y (2020) Assessing Axonal Degeneration in Embryonic Dorsal Root Ganglion Neurons In Vitro. Methods Mol Biol 2143:41-54.

Shin JE, Miller BR, Babetto E, Cho Y, Sasaki Y, Qayum S, Russler EV, Cavalli V, Milbrandt J, DiAntonio A (2012) SCG10 is a JNK target in the axonal degeneration pathway. Proc Natl Acad Sci U S A 109:E3696-3705.

Smirnova L, Harris G, Delp J, Valadares M, Pamies D, Hogberg HT, Waldmann T, Leist M, Hartung T (2016) A LUHMES 3D dopaminergic neuronal model for neurotoxicity testing allowing long-term exposure and cellular resilience analysis. Arch Toxicol 90:2725-2743.

Steiger JH (1980) Tests for Comparing Elements of a Correlation Matrix. Psychological Bulletin $87: 245-251$

Sundaramoorthy V, Green D, Locke K, O'Brien CM, Dearnley M, Bingham J (2020) Novel role of SARM1 mediated axonal degeneration in the pathogenesis of rabies. PLoS Pathog 16:e1008343.

Suzuki N, Akiyama T, Warita H, Aoki M (2020) Omics Approach to Axonal Dysfunction of Motor Neurons in Amyotrophic Lateral Sclerosis (ALS). Frontiers in neuroscience 14:194.

Tagliaferro P, Burke RE (2016) Retrograde Axonal Degeneration in Parkinson Disease. Journal of Parkinson's disease 6:1-15. 
920

921

922

923

924

925

926

927

928

929

930

931

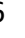

Tan VX, Lassus B, Lim CK, Tixador P, Courte J, Bessede A, Guillemin GJ, Peyrin JM (2018) Neurotoxicity of the Cyanotoxin BMAA Through Axonal Degeneration and Intercellular Spreading. Neurotox Res 33:62-75.

Wakatsuki S, Saitoh F, Araki T (2011) ZNRF1 promotes Wallerian degeneration by degrading AKT to induce GSK3B-dependent CRMP2 phosphorylation. Nat Cell Biol 13:1415-1423.

Zhai Q, Wang J, Kim A, Liu Q, Watts R, Hoopfer E, Mitchison T, Luo L, He Z (2003) Involvement of the ubiquitin-proteasome system in the early stages of wallerian degeneration. Neuron 39:217-225.

Zhang XM, Yin M, Zhang MH (2014) Cell-based assays for Parkinson's disease using differentiated human LUHMES cells. Acta Pharmacol Sin 35:945-956.

(1)

17

8

9

20

22

23

24

25

26

27 
A
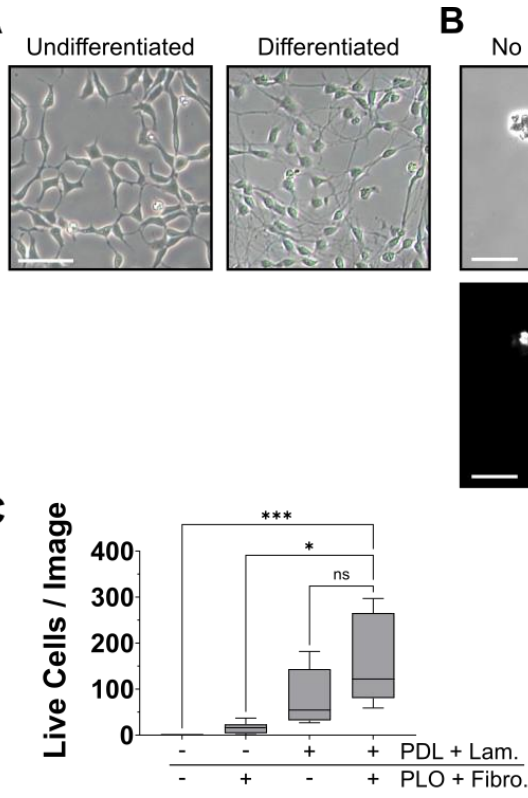

B
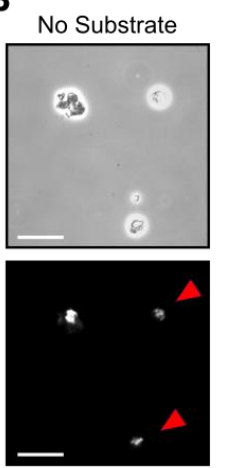

D

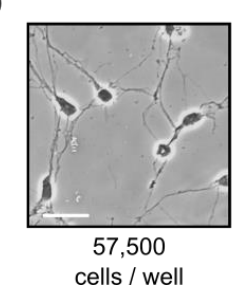

cells / well
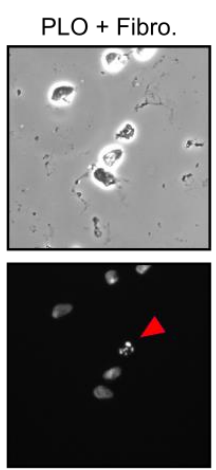

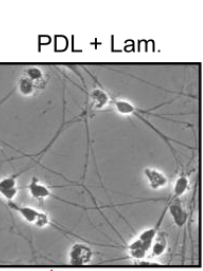

PLO + Fibro. + PDL + Lam.
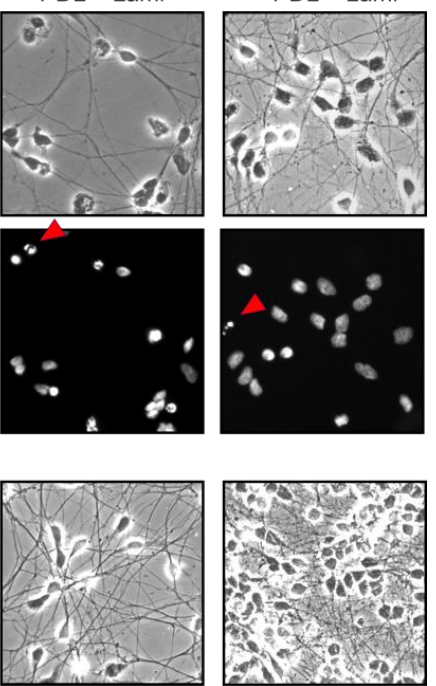

115,000

cells / well

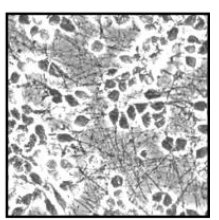

230,000

cells / well
934

Figure 1: Optimization of LUHMES Cell Culture Conditions for Measurement of Neurite Degeneration. A, representative phase contrast micrographs of LUHMES cells that were maintained in growth medium (left) or cultured in differentiation medium for five days (right). B, phase contrast micrographs (top row) or images of DAPI staining (bottom row) of LUHMES cells that were cultured in differentiation medium for five days in Lab-Tek II chamber slides that were coated as indicated with no substrate, $200 \mu \mathrm{g} / \mathrm{mL}$ poly-L-ornithine and $2 \mu \mathrm{g} / \mathrm{mL}$ fibronectin, $100 \mathrm{ng} / \mathrm{mL}$ poly-D-lysine and $10 \mu \mathrm{g} / \mathrm{mL}$ laminin, or all four substrates. Red arrowheads indicate nuclei that were considered as apoptotic due to low nuclear area or apparent nuclear fragmentation. C, quantification of live cells in cultures established as described in 1B. The cells were fixed on their fifth day of differentiation with $4 \%$ paraformaldehyde, and nuclei were labeled with 4',6'-diamidino-2-phenylindole (DAPI). Five fluorescence micrographs depicting DAPI staining were captured per condition in each experiment, and cells were scored as healthy or dying based on the appearance of nuclei. Coating with all four substrates resulted in a significant increase in the mean number of live cells compared to cultures established in slides coated with poly-L-ornithine and fibronectin $[n=6 ; p=0.0437$, Friedman test with Dunn's correction for multiple comparisons). D, representative phase contrast micrographs of LUHMES cell cultures established using different plating densities as indicated. The cells were plated in differentiation medium, and images were captured on the fifth day in culture. (Abbreviations: PLO, poly-L-ornithine; Lam.; laminin; PDL, poly-D-lysine; Fibro., Fibronectin; ${ }^{*}, \mathrm{p}<0.05$; ${ }^{* *}, \mathrm{p}<0.001 ; n$, number of experiments, each featuring an independent cell culture preparation, scalebar $=25 \mu \mathrm{m}$. 
A Phase-contrast

B

C

D

E

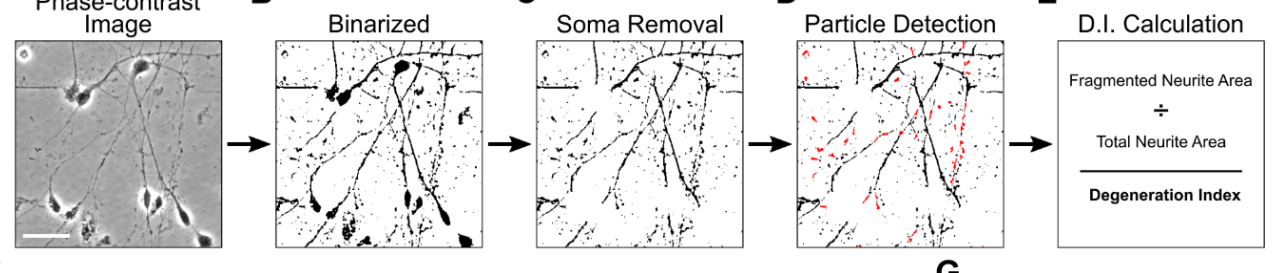

Vehicle

$2.5 \mu \mathrm{M} 6-\mathrm{OHDA}$
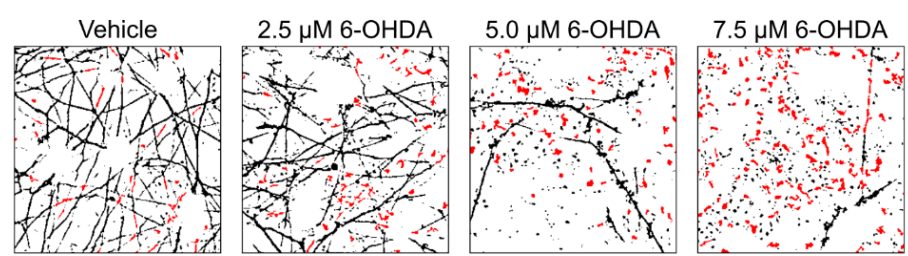

957

Figure 2: Measurement of Neurite Degeneration in LUHMES Cell Cultures Exposed to 6-OHDA using the Traditional DI Analysis Method. A - E; schematic representing the steps through which DI analyses are traditionally performed. Phase contrast images (A) are binarized to yield an image featuring black cells and white background (B). Any cell bodies in the image are then removed to generate an image exclusively featuring neurites (C). The Analyze Particles tool of ImageJ is then used to detect neurite fragments with a size of $20-10,000$ pixels and circularity of $0.2-1.0$ pixels. The detected fragments are highlighted in red (D). The summed area of the neurite fragments is divided by the total neurite area, calculating by measuring the area of all black pixels, to yield a Degeneration Index value (E). F, binarized images of LUHMES cells with cell bodies removed and neurite fragments highlighted in red. The LUHMES cells were differentiated for five days and then exposed to the indicated concentrations of 6-OHDA or vehicle solution for 24 hours. The cells were then fixed with $4 \%$ PFA, and phase contrast micrographs were subjected to $\mathrm{DI}$ analysis using the traditional procedure described in $2 \mathrm{~A}-\mathrm{E}$. G, quantification of neurite degeneration using phase contrast micrographs of LUHMES cells that were exposed to various concentrations of 6-OHDA and analyzed as described in 2F. Exposure to 6-OHDA resulted in a significant and dose-dependent increase in neurite degeneration [ $n=9$, ANOVA with Šidák correction for multiple comparisons]. Abbreviations: DI, Degeneration Index, 6-OHDA, 6-hydroxydopamine; veh, vehicle; ${ }^{* *}, p<0.01 ;{ }^{* \star * \star}, p<0.0001 ; n s$, not significant; $n$, number of experiments, each featuring an independent cell culture preparation, scalebar $=25 \mu \mathrm{m}$.

5

(1)

7

878

9


A

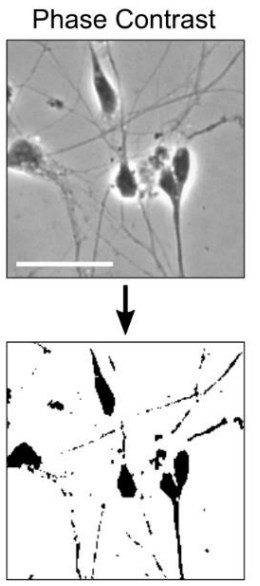

Binarized

D

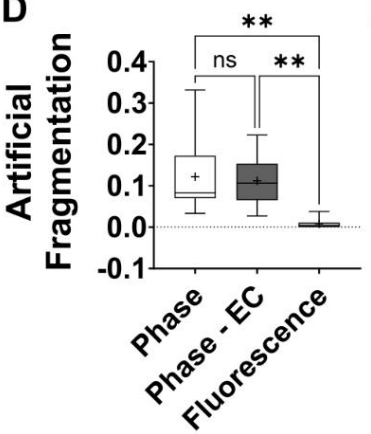

B

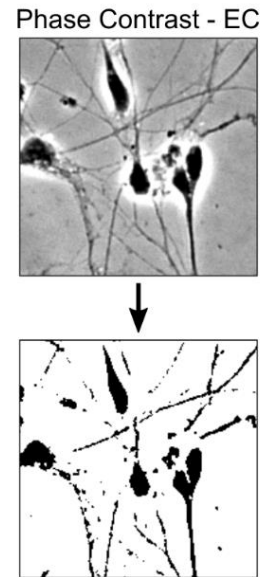

Binarized
C Fluorescence

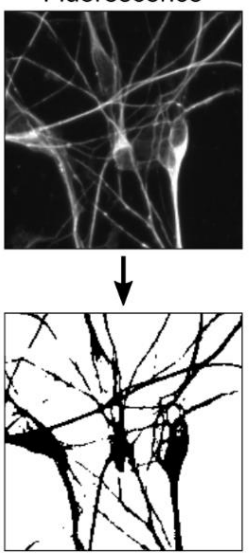

Binarized
E

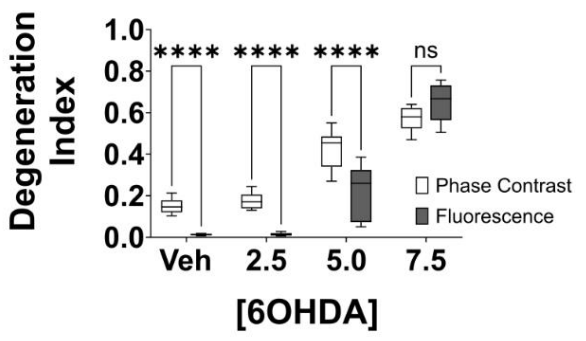

Figure 3: Reducing DI Measurement Error Associated with Binarization of Phase Contrast Micrographs. A, representative phase contrast micrograph (top) depicting LUHMES cells with intact neurites that yields an image featuring cells with fragmented neurites upon binarization (bottom). B, the phase contrast image depicted in 3A was subjected to contrast enhancement using a Lookup Table with a minimum of 90 and a maximum of 205 (top). The image was then subjected to binarization, yielding an image with comparatively greater neurite area yet still featuring fragmented neurites (bottom). C, the cells displayed in 3A and 3B were imaged by fluorescence microscopy following immunolabeling for Blll-tubulin (top). Binarization of the fluorescence micrograph yields an image featuring a higher proportion of intact neurites. D, quantification of artificial fragmentation in binarized images generated from images of healthy LUHMES cells with intact neurites. The binarized images were generated from original phase contrast images (Phase), from phase contrast images that were subjected to contrast enhancement (Phase - EC), or from fluorescence micrographs featuring an identical field of view and depicting immunofluorescence labeling for BIII-tubulin (Fluorescence). Fluorescence micrographs yielded binarized images with significantly reduced artificial fragmentation [ $n=10$ images from nine independent cell cultures; Friedman test with Dunn's correction for multiple comparisons]. E, degeneration index measurements obtained from the phase contrast micrographs generated as described in Fig. $2 \mathrm{~F}-2 \mathrm{G}$ (white bars) or images from identical fields of view depicting immunofluorescence labeling for BIII-tubulin (gray boxes). The analyses were performed using the traditional procedures described in $2 \mathrm{~A}-2 \mathrm{E}$. DI scores of cells featuring intact neurites (vehicle or $2.5 \mu \mathrm{M} 6-\mathrm{OHDA}$ treated) were significantly lower when obtained from fluorescence micrographs than when obtained from corresponding phase contrast micrographs $[n=9$ experiments featuring independent cell culture preparations, ANOVA with Šidák correction for multiple comparisons] Abbreviations: $E C$, enhanced contrast; 6-OHDA, 6-hydroxydopamine; veh, vehicle; ${ }^{* *}, \mathrm{p}<0.01 ;{ }^{* * *}, \mathrm{p}<0.0001 ; n s$, not significant, scalebar $=25 \mu \mathrm{m}$. 
A

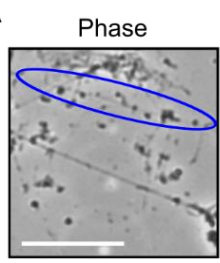

Original

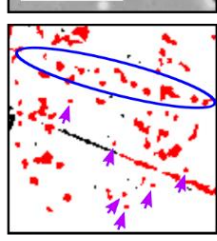

$5-10,000$

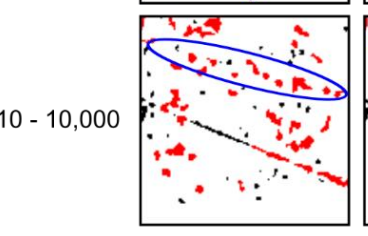

$10-10,000$
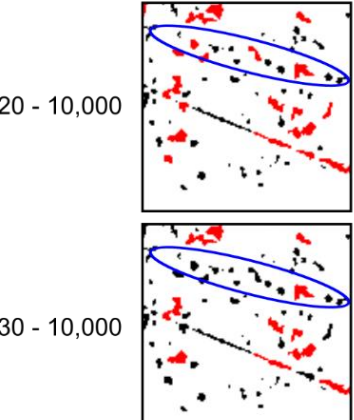

Fluorescence
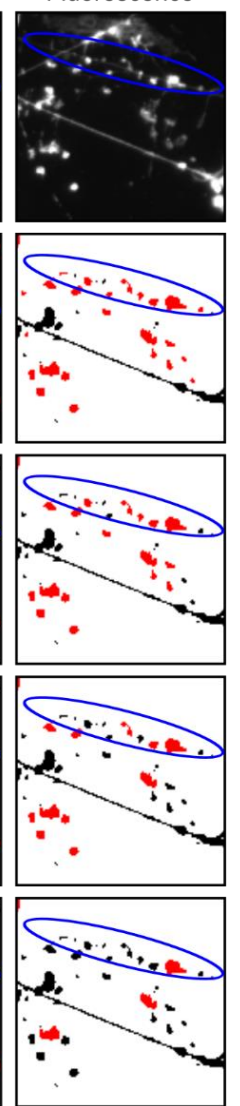

B
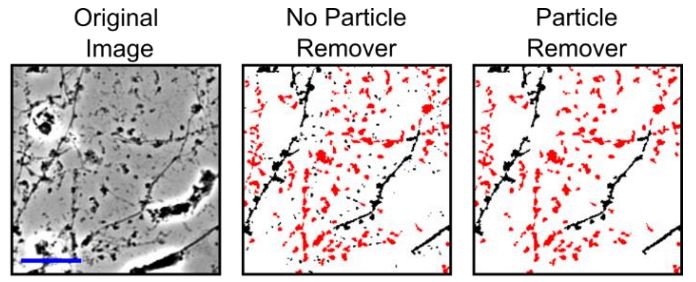

C

Phase Contrast Images

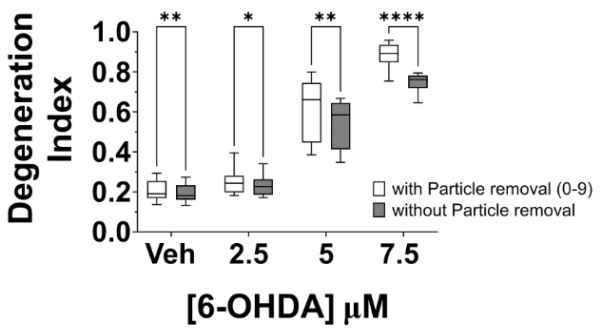

D

Fluorescence Images

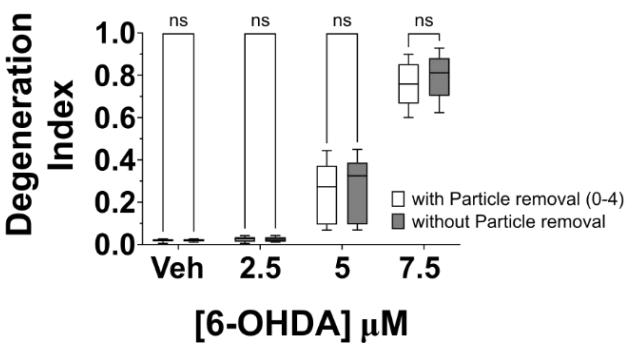

Figure 4: Refinement of Parameters Associated with Fragmented Neurite Detection. A, phase contrast (left) and fluorescence (right) micrographs depicting a fragmented neurite (circled in blue) were binarized and subjected to particle analysis using the indicated pixel size parameters for neurite fragment detection. Parameters of $5-10,000$ observably yielded the most accurate and sensitive detection of fragments in binarized images obtained from fluorescence micrograph. However, similar particle analysis parameters resulted in the erroneous detection of nonneurite debris (purple arrows) as neurite fragment in binarized images obtained from phase contrast micrographs. Thus particle analysis size parameters of $10-10,000$ pixels are recommended for DI measurements obtained from phase contrast micrographs. B, representative images depicting LUHMES cells that were exposed to $5 \mu \mathrm{M} 6-\mathrm{OHDA}$ for 24 hours. The phase contrast micrograph (left) was binarized and subjected to DI analysis using fragment size parameters of $10-10,000$ pixels. The neurite fragment detection was performed either without any additional image processing (middle image) or following removal of small particles less than 10 pixels in size (right image). C-D, quantification of neurite degeneration using phase contrast micrographs $(C)$ of LUHMES cells that were generated as described in Fig. 2F - 2G or corresponding fluorescence micrographs depicting immunofluorescence labeling for BIIItubulin (D). The analyses of phase contrast micrographs were performed using fragment size parameters of $10-$ 10,000 pixels, while fluorescence micrographs were analyzed using fragment size parameters of $5-10,000$ pixels. The analyses were performed using either binarized images without any additional image processing (white bars) or following removal of all objects smaller than neurite fragments from the image (gray bars). Removal of small particles significantly increase DI scores obtained from phase contrast images but had no significant effect on DI scores obtained from fluorescence micrographs $[n=9$ experiments featuring independent cell culture preparations, ANOVA with Šidák correction for multiple comparisons]. Abbreviations: 6-OHDA, 6-hydroxydopamine; veh, vehicle; *, $p<0.05$; ${ }^{* *}, \mathrm{p}<0.01 ;{ }^{* * * *}, p<0.0001 ; n s$, not significant; $n$, number of experiments, each featuring an independent cell culture preparation; scalebar $=20 \mu \mathrm{m}$. 


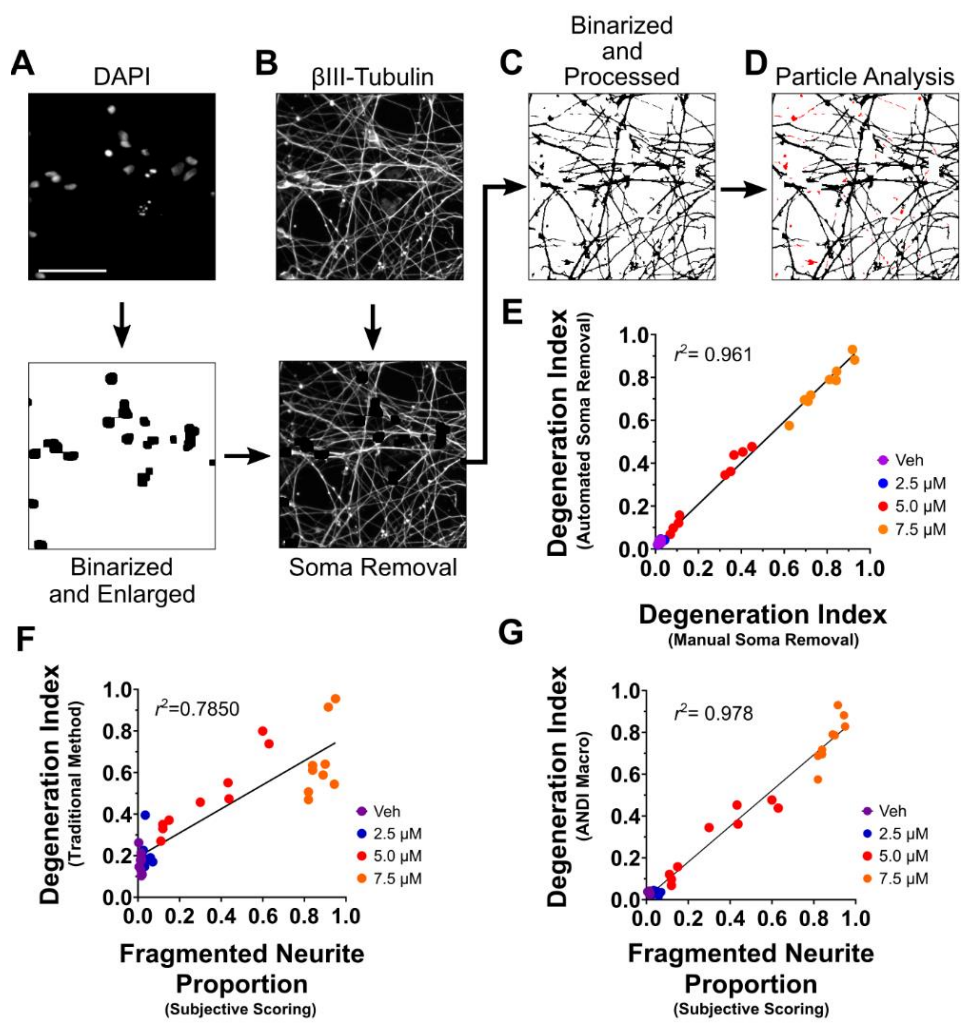

Figure 5: Accurate and Automated Measurement of Neurite Degeneration using the ANDI Macro. A-D, schematic depicting the major operations performed by the ANDI macro. Images of DAPI-stained nuclei (A, top image) are binarized and subjected to multiple dilation and erosion operations, yielding an image with black regions that have been enlarged to encompass the size of a typical cell soma ( $A$, bottom image). The image is then subtracted from a corresponding fluorescence micrograph depicting neurons immunolabeled for $\beta$ III-tubulin (B, top image), thereby producing an image exclusively featuring neurites ( $B$, bottom image). The neurite image is binarized and subjected to additional processing with the Particle Remover plugin (C). The area of black pixels is summed to calculate the total neurite area, and the Analyze Particles tool is applied to measure neurite fragments with a size between $5-10,000$ pixels (D). E, plot displaying the correlation between DI measurements obtained from images subjected to automated soma removal and DI scores from images subjected to manual soma removal. The images of LUHMES cells exposed to various concentrations of 6-OHDA, generated as described in Fig. $2 \mathrm{~F}$ and Fig. 3E were subjected to the operations of the ANDI macro related to soma removal or were subjected to manual soma removal using the freehand tool of ImageJ. The Particle Remover plugin was then applied to remove small objects less than 5 pixels in size from the images, the Measure tool was used to measure the total neurite area, and the Analyze Particles tool was applied to measure the area of fragments between $5-10,000$ pixels. DI scores obtained from images subjected to automated soma removal correlated strongly with DI measurements from images subjected to manual soma removal [ $n=36$, Spearman's rho, $\left.r(34)=0.980, r^{2}=0.961\right]$. $\mathbf{F}$, phase contrast images of LUHMES cells that were exposed to various concentrations of 6-OHDA, generated as described in Fig. 2F-2G, were subjected to blinded scoring based on the proportion of neurite area that appears fragmented, with a value of 1.0 representing $100 \%$ neurite fragmentation and a value of 0 representing no neurite fragmentation. A correlation plot depicting the subjective scores and the DI measurements obtained using traditional DI analysis procedures (as described in Fig. 2) is displayed [ $n=36$, Pearson's $\left.r=0.886, r^{2}=0.785\right]$. G, plot depicting the correlation between the subjective scores of phase contrast micrographs described in $5 \mathrm{~F}$ and $\mathrm{DI}$ measurements obtained from corresponding fluorescence micrographs using the ANDI macro. The correlation between subjective scores and DI values calculated using the ANDI macro was significantly greater than the correlation between subjective scores and DI values obtained using 
A

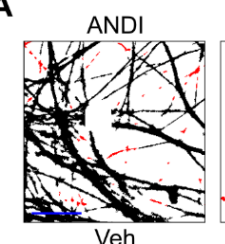

D

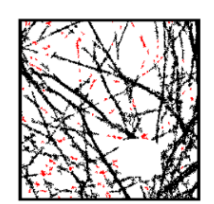

NGF+

$\mathbf{F}$

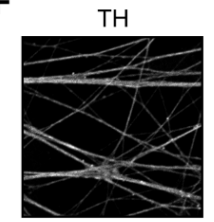

Veh

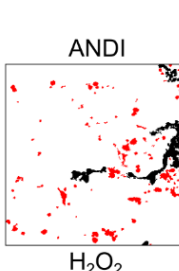

$\mathrm{H}_{2} \mathrm{O}_{2}$

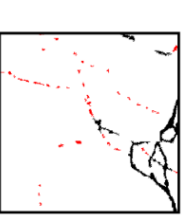

NGF -

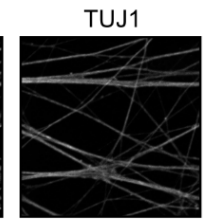

Veh
B

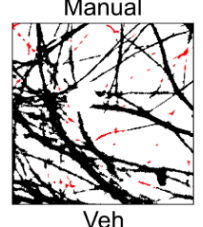

E
1060

1061

1062

1063

1064

1065

1066

1067

1068

1069

1070

1071

1072

1073

1074

1075

1076

1077

1078

1079

1080

1081

1082

1083

1084

1085

1086

1087

1088

1089
C
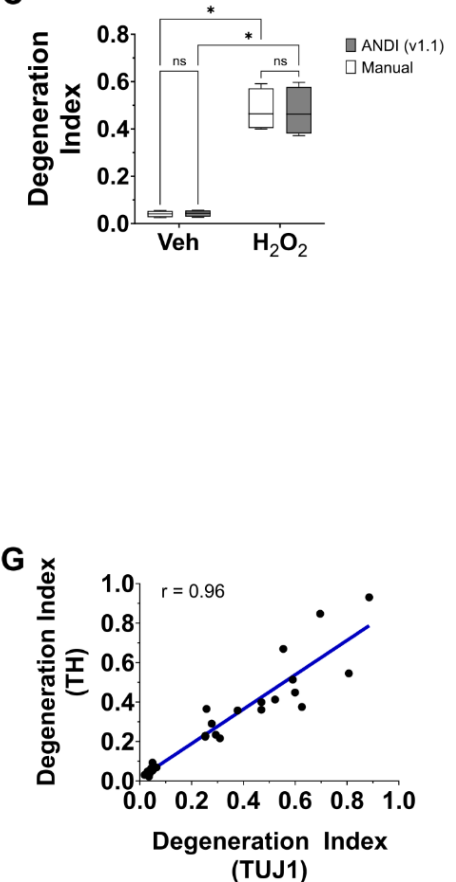

Figure 6: Utility of ANDI for Measuring of Neurite Degeneration in Primary Cultures of Sympathetic Neurons. A - B, representative images of cultured sympathetic neurons that were exposed to vehicle solution or $500 \mu \mathrm{M}$ hydrogen peroxide for 24 hours. After treatment, the neurons were fixed in $4 \%$ paraformaldehyde, immunolabeled for BIII-tubulin, and counterstained with the nucleic acid dye DAPI. Images of the cells were then either subjected to automated soma removal, binarization, and neurite fragment analysis using ANDI (A) or were subjected to manual soma removal using the freehand drawing tool of ImageJ, followed by analysis of Degeneration Index using parameters identical to those featured in ANDI (B). Detected neurite fragments are displayed in red. C, Degeneration Index scores associated with the experiments performed as described in $6 \mathrm{~A}-\mathrm{B}$. Degeneration Index scores from images of neurons exposed to hydrogen peroxide were significantly higher than those from images of neurons treated with vehicle solution ( $n=4$, ANOVA with Šidák correction for multiple comparisons, $F(1,3)=71.2, p=0.0035$, $\left.R^{2}=0.9301\right)$. No significant difference was found between degeneration index scores obtained using ANDI and those obtained following manual soma removal ( $n=4$ experiments - each featuring an independent cell culture preparation, ANOVA with Šidák correction for multiple comparisons, $\left.F(1,3)=0.1082, p=0.7639, R^{2}=0.0000\right)$. D, representative images of healthy sympathetic neuron cultures (left) or sympathetic neuron cultures subjected to NGF withdrawal for 80 hours (right). Fixed cells were immunolabeled for BIII-tubulin, counterstained with the nucleic acid dye Hoescht, and imaged by confocal microscopy. The displayed images were processed for automated soma removal, binarization, and fragment detection using ANDI. Neurite fragments are highlighted in red. E, degeneration index scores associated with the experiments described in Fig. 6D. The DI scores of neurons subjected to NGF withdrawal were significantly higher compared to DI scores from healthy cultures (repeated measures ANOVA, $n=3$ experiments - each featuring an independent cell culture preparation, $\mathrm{F}(1,2)=287, \mathrm{p}=0.0035, R^{2}=0.958$,). $\mathrm{F}$, representative images of healthy, vehicle-treated sympathetic neuron cultures or degenerating sympathetic neuron cultures treated with $500 \mu \mathrm{M}$ hydrogen peroxide. After a 24 hour treatment period, the cells were fixed with $4 \%$ paraformaldehyde in PBS and immunolabeled for tyrosine hydroxylase (TH) or Blll-tubulin (TUJ1). Counterstaining was performed with the nucleic acid dye DAPI (not shown). G, plot displaying the correlation between DI scores generated by ANDI from images depicting tyrosine hydroxylase staining (y-axis) and from micrographs of BIII-tubulin staining (x-axis). Measurements were obtained from images of sympathetic neurons that were treated for 24 hours with vehicle solution, $100 \mu \mathrm{M}$ hydrogen peroxide, or $500 \mu \mathrm{M}$ hydrogen peroxide, followed by fixation with $4 \%$ paraformaldehyde, immunolabeling for tyrosine hydroxylase and BIII-tubulin, and counterstaining with DAPI. A strong correlation was observed between DI scores from images of tyrosine hydroxylase staining and DI scores from images 
of Blll-tubulin staining (Spearman's rho $=0.96, \mathrm{n}=30$ images from three independent cell culture preparations). Abbreviations: Veh, vehicle; NGF+, incubated in media containing nerve growth factor; NGF-, incubated in media lacking nerve growth factor; ${ }^{*}, \mathrm{p}<0.05 ;{ }^{* *}, \mathrm{p}<0.01 ; n s$, not significant; scalebar, $25 \mu \mathrm{m}$.

A

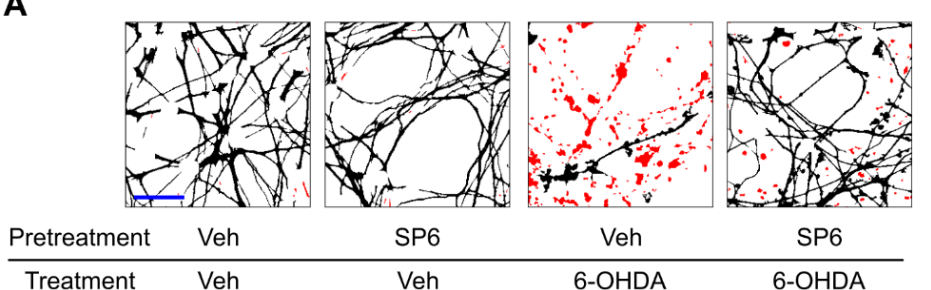

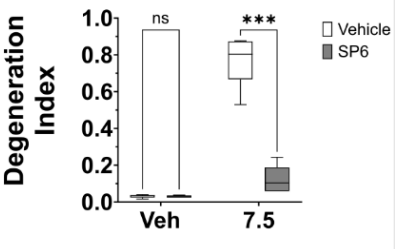

[6-OHDA] $\mu \mathrm{M}$

Figure 7: Use of the ANDI Macro to Investigate the Effects of JNK Signaling on Oxidative Stress-Induced Neurite Degeneration in Human Mesencephalic Cells. A, representative images of differentiated LUHMES cells that were exposed for 24 hours to vehicle solution or $7.5 \mu \mathrm{M} 6$-OHDA following 1-hour pretreatment with $10 \mu \mathrm{M}$ SP600125 or vehicle solution. Fixed cells were immunolabeled for Blll-tubulin, and fluorescence micrographs were analyzed using the ANDI macro. Representative images that were processed for automated soma removal, binarization, and fragment detection are displayed. B, degeneration index scores associated with the experiments described in Fig. 6A. Pretreatment with the JNK inhibitor SP600125 resulted in a significant decrease in neurite degeneration induced by 6 -OHDA $(n=6$, ANOVA with Šidák correction for multiple comparisons]. Abbreviations: 6 $O H D A$, 6-hydroxydopamine; veh, vehicle; ${ }^{\star \star *}, \mathrm{p}<0.001 ; n s$, not significant; $n$, number of experiments, each featuring an independent cell culture preparation; scalebar $=25 \mu \mathrm{m}$. 
Table-1: Statistical Table

\begin{tabular}{|c|c|c|c|c|c|}
\hline Data & $\begin{array}{c}\text { Data } \\
\text { Structure }\end{array}$ & Type of Test & \multicolumn{3}{|c|}{$\begin{array}{c}\text { Power } \\
\text { (95\% Confidence Intervals) }\end{array}$} \\
\hline Fig. 1C & Non-normal & $\begin{array}{l}\text { Friedman test with } \\
\text { Dunn's correction }\end{array}$ & \multicolumn{3}{|l|}{ Not Applicable } \\
\hline \multirow[t]{6}{*}{ Fig. 2G } & \multirow[t]{6}{*}{ Normal } & \multirow{6}{*}{$\begin{array}{l}\text { ANOVA with } \\
\text { Šidák correction }\end{array}$} & \multicolumn{2}{|l|}{$0 \mu \mathrm{M}$ (Vehicle) vs. $2.5 \mu \mathrm{M} 6-\mathrm{OHDA}$} & -0.06461 to 0.01985 \\
\hline & & & \multirow{2}{*}{\multicolumn{2}{|c|}{$\begin{array}{l}0 \mu \mathrm{M} \text { (Vehicle) vs. } 5.0 \mu \mathrm{M} \text { 6-OHDA } \\
0 \mu \mathrm{M} \text { (Vehicle) vs. } 7.5 \mu \mathrm{M} \text { 6-OHDA }\end{array}$}} & -0.3547 to -0.1754 \\
\hline & & & & & -0.5079 to -0.3277 \\
\hline & & & \multicolumn{2}{|c|}{$2.5 \mu \mathrm{M} 6-\mathrm{OHDA}$ vs. $5.0 \mu \mathrm{M} 6-\mathrm{OHDA}$} & -0.3429 to -0.1424 \\
\hline & & & \multicolumn{2}{|l|}{$2.5 \mu \mathrm{M} 6-\mathrm{OHDA}$ vs. $7.5 \mu \mathrm{M}$ 6-OHDA } & -0.4865 to -0.3044 \\
\hline & & & \multicolumn{2}{|c|}{$5.0 \mu \mathrm{M}$ 6-OHDA vs. $7.5 \mu \mathrm{M}$ 6-OHDA } & -0.2632 to -0.04233 \\
\hline Fig. 3D & Non-normal & $\begin{array}{l}\text { Friedman test with } \\
\text { Dunn's correction }\end{array}$ & \multicolumn{3}{|l|}{ Not Applicable } \\
\hline \multirow[t]{4}{*}{ Fig. 3E } & \multirow[t]{4}{*}{ Normal } & \multirow{4}{*}{$\begin{array}{l}\text { ANOVA with } \\
\text { Šidák correction }\end{array}$} & \multirow{2}{*}{\multicolumn{2}{|c|}{$\begin{array}{l}0 \mu \mathrm{M} \text { (Vehicle) - Phase vs Fluorescence } \\
2.5 \mu \mathrm{M} 6 \mathrm{OHDA} \text { - Phase vs Fluorescence }\end{array}$}} & 0.1055 to 0.1741 \\
\hline & & & & & 0.1213 to 0.1985 \\
\hline & & & \multicolumn{2}{|c|}{$5.0 \mu \mathrm{M} 6 \mathrm{OHDA}$ - Phase vs Fluorescence } & 0.1462 to 0.2764 \\
\hline & & & \multicolumn{2}{|c|}{$7.5 \mu \mathrm{M} 6 \mathrm{OHDA}$ - Phase vs Fluorescence } & -0.1731 to 0.003029 \\
\hline \multirow[t]{4}{*}{ Fig. 4C } & \multirow[t]{4}{*}{ Normal } & \multirow[t]{4}{*}{$\begin{array}{l}\text { ANOVA with } \\
\text { Šidák correction }\end{array}$} & \multicolumn{2}{|c|}{$\begin{array}{l}0 \mu \mathrm{M} \text { (Vehicle) - With Particle Removal } \\
\text { vs Without Particle Removal }\end{array}$} & 0.005456 to 0.02108 \\
\hline & & & \multicolumn{2}{|c|}{$\begin{array}{l}2.5 \mu \mathrm{M} \text { 6OHDA - With Particle Removal } \\
\text { vs Without Particle Removal }\end{array}$} & 0.004059 to 0.03360 \\
\hline & & & \multicolumn{2}{|c|}{$\begin{array}{l}5.0 \mu \mathrm{M} 6 \mathrm{OHDA} \text { - With Particle Removal } \\
\text { vs Without Particle Removal }\end{array}$} & 0.03510 to 0.1167 \\
\hline & & & \multicolumn{2}{|c|}{$\begin{array}{l}7.5 \mu \mathrm{M} \text { 6OHDA - With Particle Removal } \\
\text { vs Without Particle Removal }\end{array}$} & 0.09786 to 0.1797 \\
\hline \multirow[t]{4}{*}{ Fig. 4D } & \multirow[t]{4}{*}{ Normal } & \multirow[t]{4}{*}{$\begin{array}{l}\text { ANOVA with } \\
\text { Šidák correction }\end{array}$} & \multicolumn{2}{|c|}{$\begin{array}{l}0 \mu \mathrm{M} \text { (Vehicle) - With Particle Removal } \\
\text { vs Without Particle Removal }\end{array}$} & -0.002760 to 0.001501 \\
\hline & & & \multicolumn{2}{|c|}{$\begin{array}{l}2.5 \mu \mathrm{M} 6 \mathrm{OHDA} \text { - With Particle Removal } \\
\text { vs Without Particle Removal }\end{array}$} & -0.003088 to 0.002654 \\
\hline & & & \multicolumn{2}{|c|}{$\begin{array}{l}5.0 \mu \mathrm{M} 6 \mathrm{OHDA} \text { - With Particle Removal } \\
\text { vs Without Particle Removal }\end{array}$} & -0.04534 to 0.01876 \\
\hline & & & $\begin{array}{l}7.5 \mu \mathrm{M} \text { 6OHDA - With Particle Re } \\
\text { vs Without Particle Removal }\end{array}$ & noval & -0.1022 to 0.02869 \\
\hline Fig. 5E & Non-normal & Spearman's rho & 0.9604 to 0.9901 & & \\
\hline Fig. 5F & Normal & Pearson's $r$ & 0.786 to 0.941 & & \\
\hline Fig. 5G & Normal & Pearson's $r$ & 0.978 to 0.994 & & \\
\hline Fig. $6 \mathrm{C}$ & Normal & $\begin{array}{l}\text { ANOVA with } \\
\text { Šidák correction }\end{array}$ & $\begin{array}{l}\text { Veh:ANDI (v1.1) vs. } \\
\text { Veh:Manual }\end{array}$ & -0.0005 & 736 to 0.004670 \\
\hline & & & $\begin{array}{l}\mathrm{H} 2 \mathrm{O} 2: \mathrm{ANDI}(\mathrm{v} 1.1) \text { vs. } \\
\text { H2O2:Manual }\end{array}$ & -0.0860 & 3 to 0.07370 \\
\hline & & & $\begin{array}{l}\text { Veh:ANDI (v1.1) vs. } \mathrm{H}_{2} \mathrm{O}_{2}: \text { ANDI } \\
\text { (v1.1) }\end{array}$ & -0.7698 & to -0.09204 \\
\hline & & & Veh:Manual vs. $\mathrm{H}_{2} \mathrm{O}_{2}$ :Manual & -0.742 & to -0.1360 \\
\hline Fig. 6E & Normal & $\begin{array}{l}\text { Repeated } \\
\text { Measures ANOVA }\end{array}$ & 0.04244 to 0.07132 & & \\
\hline Fig. 6G & Non-normal & Spearman's rho & 0.9186 to 0.9822 & & \\
\hline Fig. 7B & Normal & $\begin{array}{l}\text { ANOVA with } \\
\text { Šidák correction }\end{array}$ & $\begin{array}{l}0 \mu \mathrm{M} \text { (Vehicle) - Pretreatment wit } \\
\text { Vehicle vs SP } 600125\end{array}$ & & -0.006446 to 0.01094 \\
\hline & & & $\begin{array}{l}7.5 \mu \mathrm{M} \text { 6OHDA - Pretreatment wi } \\
\text { Vehicle vs SP600125 }\end{array}$ & & 0.4796 to 0.8076 \\
\hline
\end{tabular}

Table-1: Table indicating the data structure (normal or non-normal distribution), type of statistical test, and 95\% confidence intervals associated with the experiments described in this article. 


\section{Extended Data 1}

1129

setBatchMode(true);

1130 //identify directories and create file lists

1131 NeuriteDir = getDirectory("Choose folder with neurite staining"); SaveDir = getDirectory("Choose Where to Save");

NeuriteList $=$ getFileList(NeuriteDir);

1135 DapiList = getFileList(DapiDir);

1136 if (NeuriteList.length != DapiList.length) \{exit("number of images in two directories is not equal")\};

1137 $p=$ getNumber("Dilate the nuclei how many times? (Default is 12)", 12);

1138 run("Clear Results");

1139 close("*");

1140 //initiate for loop

1141 for $(f=0 ; f<$ NeuriteList.length; $f++)\{$

1142

1143

1144

1145

1146

1147

1148

1149

1150

1151

1152

1153

1154

1155

1156

1157

1158

1159

1160

1161

1162

1163

1164

1165

1166

1167

1168

1169

//code to prevent errors due to Desktop.ini files

IniFilelndex = indexOf(DapiDir + DapiList[f],".ini");

if(IniFilelndex $>=0)$ print("skipped due to .ini file detection"); continue;\}

open(DapiDir + DapiList[f]);

OriDapilmage $=$ getTitle () ;

run("8-bit");

run("Set Scale...", "distance=0 known=0 pixel=1 unit=pixel");

//run("Brightness/Contrast...");

setMinAndMax (10, 245);

run("Apply LUT");

//make binary and remove particles

run("Colors...", "foreground=white background=black selection=yellow");

setOption("BlackBackground", false);

run("Options...", "iterations=1 count=1");

run("Make Binary");

run("Particle Remover", "size=0-10");

close(OriDapilmage);

//dilate and erode

setOption("BlackBackground", false);

for $(d=0 ; d<p ; d++)\{$ run("Dilate");

\}

run("Erode");

run("Erode");

run("Erode");

run("Erode");

run("Erode");

rename("Y"+f);

//open neurite image

open(NeuriteDir + NeuriteList[f]);

OriNeuritelmage = getTitle();

//convert image to 8-bit and remove any scale information run("8-bit");

run("Set Scale...", "distance=0 known=0 pixel=1 unit=pixel");

//contrast enhancement 
setMinAndMax(0, 195);

run("Apply LUT");

print(" Cell body removal for " + OriNeuritelmage + "...");

//image subtraction

imageCalculator("Subtract create", OriNeuritelmage,"Y"+f);

//close old images and temporarily rename image with cell bodies removed close(OriNeuritelmage);

close $(" Y "+f)$;

rename("X" + f);

print(" Binarization of " + OriNeuritelmage + "...");

//make binary and remove particles

setOption("BlackBackground", false);

run("Options...", "iterations=1 count=1");

run("Make Binary");

run("Particle Remover", "size=0-4");

close $(" X "+f)$;

//rename image

periodindex = indexOf(OriNeuritelmage, ".");

if $($ periodindex $>=0$ ) OriNeuritelmage $=$ substring(OriNeuritelmage, 0 , periodindex); rename(OriNeuritelmage + "_" + "result");

//take measurements

print(" Analyzing " + OriNeuritelmage + "...");

setOption("BlackBackground", false);

run("Make Binary");

run("Set Measurements...", "area limit display redirect=None decimal=3");

run("Measure"); summarize");

run("Analyze Particles...", "size=5-10000 circularity=0.20-1.00 show=[Overlay Masks]

//save images

selectWindow(OriNeuritelmage + " _" + "result");

run("Flatten");

saveAs("tiff", SaveDir + OriNeuritelmage + " _" + "result");

close(OriNeuritelmage + "_" + "result");

print(" Analysis of " + OriNeuritelmage + " complete"); \}

IJ.renameResults("Summary", "Fragmented Neurite Area");

IJ.renameResults("Results", "Total Neurite Area");

selectWindow("Fragmented Neurite Area");

saveAs("Results", SaveDir +"Fragmented_Neurite_Area.csv");

selectWindow("Total Neurite Area");

saveAs("Results", SaveDir + "Total_Neurite_Area.csv");

print("Results have been saved in the selected directory.");

//save result

//saveAs("results", "C:IU sers||kraemerb||Desktop||Dapi Test Folder|\Dir3")

Extended Data 1: ANDI v1.1 script for image processing and DI analysis using ImageJ. 